\title{
Type and cotype of operator spaces
}

\author{
by
}

Hun Hee Lee (Waterloo, Ont.)

\begin{abstract}
We consider two operator space versions of type and cotype, namely $S_{p^{-}}$ type, $S_{q}$-cotype and type $(p, H)$, cotype $(q, H)$ for a homogeneous Hilbertian operator space $H$ and $1 \leq p \leq 2 \leq q \leq \infty$, generalizing " $O H$-cotype 2" of G. Pisier. We compute type and cotype of some Hilbertian operator spaces and $L_{p}$ spaces, and we investigate the relationship between a homogeneous Hilbertian space $H$ and operator spaces with cotype $(2, H)$. As applications we consider operator space versions of generalized little Grothendieck's theorem and Maurey's extension theorem in terms of these new notions.
\end{abstract}

1. Introduction. Type and cotype play an important role in Banach space theory. Thus, it is natural to expect operator space analogues of type and cotype. Actually there has been several attempts to define type and cotype in the operator space category. In [18] G. Pisier defined $O H$-cotype 2 and M. Junge (Chapter 4 of [8]) studied a variant of this notion, namely cotype $(2, R+C)$, where $O H$ and $R+C$ are the operator Hilbert space and the operator space sum of the row and column Hilbert spaces, respectively. In this paper we are going to give two different definitions of type and cotype of operator spaces, namely $S_{p}$-type, $S_{q}$-cotype and type $(p, H)$, cotype $(q, H)$ for a homogeneous Hilbertian operator space $H$, which are both generalizations of Pisier's "OH-cotype 2".

In order to get a satisfactory theory we need to focus on two aspects. The first one is about how big the cotype 2 class is. Note that the cotype 2 class in the Banach space category includes all (noncommutative) $L_{p}$ $(1 \leq p \leq 2)$ spaces. The second one is about the possibility of applications such as generalized little Grothendieck's theorem, Maurey's extension theorem and Kwapien's theorem. Although these new notions still do not promise satisfactory results in both aspects, each definition has its own pros. For example, type $(p, H)$ and cotype $(q, H)$ of $L_{p}$ spaces behave well for some good

2000 Mathematics Subject Classification: Primary 47L25; Secondary 46 B07.

Key words and phrases: completely summing maps, operator space, cotype.

This work was supported by the Korea Research Foundation Grant funded by the Korean Government(MOEHRD) (KRF- 2005-214-C00176). 
choice of $H$, and both notions allow corresponding applications mentioned above. Moreover, cotype $(2, H)$ gives us an insight about the relationship between a homogeneous Hilbertian space $H$ and an operator space $E$ which has cotype $(2, H)$. More precisely, it is known $([8,18])$ that $S_{1}$ (the trace class on $\left.\ell_{2}\right)$ has cotype $(2, R+C)$ but not cotype $(2, O H)$. Thus, it is natural to be interested in which $H$ is best among all $H$ for which $S_{1}$ has cotype $(2, H)$. This question will be answered later for all $L_{p}(1 \leq p \leq 2)$ spaces and the meaning of "best" will be clarified.

Now let us discuss our approach more precisely. Recall that a Banach space $X$ is said to have (gaussian) type $p(1 \leq p \leq 2)$ if there exists a constant $C>0$ such that

$$
\pi_{p^{\prime}, 2}\left(v^{*}\right) \leq C \cdot \ell^{*}(v)
$$

for $1 / p+1 / p^{\prime}=1$ and every $v: X \rightarrow \ell_{2}^{n}, n \in \mathbb{N}$, and (gaussian) cotype $q$ $(2 \leq q \leq \infty)$ if there exists a constant $C^{\prime}>0$ such that

$$
\pi_{q, 2}(u) \leq C^{\prime} \cdot \ell(u)
$$

for every $u: \ell_{2}^{n} \rightarrow X$ and $n \in \mathbb{N}$. Here, $\pi_{q, 2}(\cdot)$ is the $(q, 2)$-summing norm defined by

$$
\pi_{q, 2}(T: X \rightarrow Y)=\sup \left\{\frac{\left(\sum_{k}\left\|T x_{k}\right\|^{q}\right)^{1 / q}}{\left\|\sum_{k} x_{k} \otimes e_{k}\right\|_{X \otimes_{\lambda} \ell_{2}}}\right\}
$$

where $\otimes_{\lambda}$ is the injective tensor product of Banach spaces, and $\ell(u)$ and $\ell^{*}(v)$ are the $\ell$-norm and adjoint $\ell$-norm, respectively, defined by

$$
\ell(u):=\left[\int_{\Omega}\left\|\sum_{k=1}^{n} g_{k}(\omega) u e_{k}\right\|_{X}^{2} d P(\omega)\right]^{1 / 2}
$$

for i.i.d. gaussian variables $\left\{g_{k}\right\}$ on a probability space $(\Omega, P)$ and

$$
\ell^{*}(v):=\sup \left\{\operatorname{tr}(v u) \mid u: \ell_{2}^{n} \rightarrow X, \ell(u) \leq 1\right\} .
$$

Pisier's definition of $O H$-cotype 2 is as follows. An operator space $E$ is said to have $O H$-cotype 2 if there is a constant $C>0$ such that for all $v: E \rightarrow \ell_{2}^{n}$,

$$
\ell^{*}(v) \leq C \pi_{2, o h}(v)
$$

where $\pi_{2, o h}(v)$ is the $(2, o h)$-summing norm defined by

$$
\pi_{2, o h}(v)=\sup \left\{\frac{\left(\sum_{k}\left\|v x_{k}\right\|^{2}\right)^{1 / 2}}{\left\|\sum_{k} x_{k} \otimes e_{k}\right\|_{E \otimes_{\min } O H}}\right\}
$$

and $\otimes_{\min }$ is the injective tensor product of operator spaces. Note that this definition is based on the trace dual formulation of (1.2). Thus, in order to extend these notions to general exponents $q \geq 2$ we need to consider the trace dual version of (1.3). However, unlike the Banach space case we 
have the problem that $\pi_{2, o h}$ is not self-dual. We can resolve this difficulty by observing that (Proposition 6.2 in [21])

$$
\pi_{2, o h}\left(v: E \rightarrow \ell_{2}^{n}\right)=\pi_{2}^{o}\left(v: E \rightarrow O H_{n}\right),
$$

where $\pi_{2}^{o}(\cdot)$ is the completely 2 -summing norm defined by

$$
\pi_{2}^{o}(T: E \rightarrow F)=\sup \left\{\frac{\left\|\left(T x_{i j}\right)\right\|_{S_{2}(F)}}{\left\|\sum_{i, j} x_{i j} \otimes e_{i j}\right\|_{E \otimes_{\min } S_{2}}}\right\}
$$

and $S_{r}(F)(1 \leq r \leq \infty)$ is the vector-valued Schatten class introduced in [21]. Then since $\pi_{2}^{o}$ is self-dual (which will be checked later) we can reformulate (1.3) as follows. $E$ has $O H$-cotype 2 if there is a constant $C>0$ such that for all $u: O H_{n} \rightarrow E$, we have

$$
\pi_{2}^{o}(u) \leq C \cdot \ell(u) .
$$

Now it is easy to extend the cotype notion to the $q \geq 2$ case by replacing $\pi_{2}^{o}(u)$ with the completely $(q, 2)$-summing norm $\pi_{q, 2}^{o}(u)$ defined by

$$
\pi_{q, 2}^{o}(T: E \rightarrow F):=\sup \left\{\frac{\left\|\left(T x_{i j}\right)\right\|_{S_{q}(F)}}{\left\|\sum_{i, j} x_{i j} \otimes e_{i j}\right\|_{E \otimes_{\min } S_{2}}}\right\}
$$

and we will call it $S_{q}$-cotype. $S_{p^{-}}$type can be defined similarly.

There is another approach using approximation numbers. This can be done in a more general context. Let $H$ be a homogeneous Hilbertian operator space, i.e. $H$ is isometric to a Hilbert space and for every $u: H \rightarrow H$ we have $\|u\|_{\mathrm{cb}}=\|u\|$. Then we can define $\pi_{2, H}(v)$ by replacing $O H$ with $H$ and use it in the definition of cotype $(2, H)$. In order to ensure that $\pi_{2, H}(\cdot)$ is actually a norm we need to assume that $H$ is "subquadratic", i.e. for all orthogonal projections $\left\{P_{i}\right\}_{i=1}^{n}$ in $H$ with $I_{H}=P_{1}+\cdots+P_{n}$ we have

$$
\|x\|_{B\left(\ell_{2}\right) \otimes_{\min } H}^{2} \leq \sum_{i=1}^{n}\left\|I_{B\left(\ell_{2}\right)} \otimes P_{i}(x)\right\|_{B\left(\ell_{2}\right) \otimes_{\min } H}^{2}
$$

for any $x \in B\left(\ell_{2}\right) \otimes H$ (see p. 82 of [20]).

$E$ is said to have cotype $(2, H)$ if there is a constant $C>0$ such that for all $u: \ell_{2}^{n} \rightarrow E$, we have

$$
\pi_{2, H}^{*}(u) \leq C \cdot \ell(u),
$$

where $\pi_{2, H}^{*}$ is the trace dual of $\pi_{2, H}$. Now we recall the equivalence between $\pi_{q, 2}(u)$ and $\left(\sum_{k} a_{k}(u)^{q}\right)^{1 / q}$ for $u: \ell_{2} \rightarrow X$ (Corollary 19.7 of [26]) where $a_{k}(\cdot)$ is the $k$ th approximation number defined by

$$
a_{k}(u)=\inf \left\{\|u-v\|: v \in B\left(\ell_{2}, X\right), \operatorname{rk}(v)<k\right\} .
$$

Since we do not have an appropriate $(q, 2)$-extension of $\pi_{2, H}^{*}$ we use the $\ell_{q}$-sum of cb (completely bounded) approximation numbers of the map $u$ : $H_{n}^{*} \rightarrow E$, where $H_{n}^{*}$ is the $n$-dimensional version of $H^{*}$. See Section 3 for the 
details. We will call it cotype $(q, H)$, and type $(p, H)$ can be defined similarly. Note that $S_{2}$-type and $S_{2}$-cotype are equivalent to type $(2, O H)$ and cotype $(2, O H)$, respectively.

The behavior of $S_{q}$-cotype of $L_{p}$ spaces is quite different from the Banach space case. Moreover, the behavior of cotype $(2, H)$ depends on $H$. More precisely, we have the following.

Theorem. Let $1 \leq p \leq 2$ and $\mu$ be a $\sigma$-finite measure.

(1) $S_{p}$ has cotype $(2, H)$ if and only if the formal identity

$$
\text { id : } R C[p] \rightarrow H
$$

is completely bounded.

(2) $L_{p}(\mu)$ has cotype $(2, H)$ if and only if the formal identity

$$
\text { id : } R C\left[p^{\prime}\right] \rightarrow H
$$

is completely bounded.

Note that $R C[r]=[R \cap C, R+C]_{1 / r}$ for $1 \leq r \leq \infty$, where $\cap$ and $[\cdot, \cdot]_{1 / r}$ are the intersection and the complex interpolation space in the category of operator spaces, respectively. Thus we can say that $R C[p]$ (resp. $R C\left[p^{\prime}\right]$ ) is the best (in the above sense) homogeneous Hilbertian operator space $H$ for which $S_{p}$ (resp. $\left.L_{p}(\mu)\right)$ has cotype $(2, H)$.

With these notions of type and cotype we can consider several applications. The first one is an operator space analogue of "generalized little Grothendieck's theorem" (see [3, 13] for the Banach space case).

TheOREM. For any compact set $K$ and $2 \leq q<\infty$, every bounded linear map from $C(K)$ into an $S_{q}$-cotype space is completely $(q, 2)$-summing.

The second one is an operator space analogue of "Maurey's extension theorem" (see [14] for the Banach space case). We say that an operator space $H$ is perfectly Hilbertian if $H$ is a homogeneous Hilbertian operator space and $H$ and $H^{*}$ are subquadratic (see Section 8 of [20]). Note that $R[p]=[R, C]_{1 / p}$ and $C[p]=[C, R]_{1 / p}$ are perfectly Hilbertian, and $R C[p]$ is completely isomorphic to a perfectly Hilbertian operator space. Then we have the following.

TheOREM. Let $E$ and $F$ be operator spaces of type $(2, H)$ and cotype $\left(2, H^{*}\right)$, respectively, for a perfectly Hilbertian operator space $H$. Then there is a constant $C>0$ such that for any subspace $G \subseteq E$ and any bounded linear map $u: G \rightarrow F$ we have an extension $\widetilde{u}: E \rightarrow F$ with

$$
\gamma_{H}(\widetilde{u}) \leq C\|u\| .
$$

Recall that $\gamma_{H}(\widetilde{u})=\inf \left\{\|A\|_{\mathrm{cb}}\|B\|_{\mathrm{cb}}\right\}$, where the infimum is taken over all possible factorizations $\widetilde{u}: E \stackrel{A}{\rightarrow} H(I) \stackrel{B}{\rightarrow} F$ for some index set $I$. We need "perfectness" of $H$ to ensure that $\gamma_{H}(\cdot)$ is actually a norm. By the Remark 
on p. 82 of [20], $H(I)$ is well defined for any index set $I$. As a corollary we get operator space versions of "Kwapień's theorem". See [10] for the classical Banach space case and [6] for another operator space case.

Note that there is a different notion of type and cotype of operator spaces by J. García-Cuerva and J. Parcet using quantized orthonormal systems $([5,6,16])$. At the end of Section 2 we will see how $S_{2}$-type and $S_{2}$-cotype are related to the type 2 and cotype 2 of [6].

This paper is organized as follows. In Section 2, we define $S_{p}$-type and $S_{q}$-cotype of operator spaces and develop their basic theory. As examples, we estimate $S_{p}$-type and $S_{q}$-cotype of $R[p]=[R, C]_{1 / p}, C[p]=[C, R]_{1 / p}$ and of $L_{p}$ spaces. $S_{p}$-type and $S_{q}$-cotype of commutative $L_{p}$ spaces are the same as in the Banach space case while those of $S_{p}$ are completely different. In Section 3 we define type $(p, H)$ and cotype $(q, H)$ of operator spaces and investigate their basic properties. As examples, we compute type $(p, H)$ and cotype $(q, H)$ of $R[p], C[p]$ and of $L_{p}$ spaces. Note that we can recover the same result as in the Banach space case for $S_{p}$ with a "good" choice of $H$. Moreover, we investigate the relationship between a homogeneous Hilbertian space $H$ and operator spaces of cotype $(2, H)$. In the last section we present the above two applications of type and cotype notions of this paper.

Throughout this paper, we will assume some knowledge of operator space theory $([4,22])$, completely $p$-summing maps ([21]), absolutely $p$-summing operators $([2,26])$ and vector-valued noncommutative $L_{p}$ spaces $([21])$. For $1 \leq p \leq \infty, S_{p}\left(\right.$ resp. $\left.S_{p}^{n}\right)$ and $S_{p}(E)\left(\right.$ resp. $\left.S_{p}^{n}(E)\right)$ are the Schatten classes on $\ell_{2}$ (resp. $\left.\ell_{2}^{n}\right)$ and their vector-valued versions ([21]). We will assume that all $L_{p}$ spaces (commutative or not) and their vector-valued versions are endowed with their natural operator space structure in the sense of [21]. In this paper $H$ is reserved for a homogeneous Hilbertian operator space on $\ell_{2}$; we will denote its $n$-dimensional version by $H_{n}$. In particular, $R_{n}[p], C_{n}[p]$ and $R C_{n}[p]$ are $n$-dimensional versions of $R[p], C[p]$ and $R C[p]$ respectively. As usual, $B(E, F)$ and $C B(E, F)$ denote the sets of all bounded linear maps and all completely bounded linear maps from $E$ into $F$, respectively. We use the symbol $a \lesssim b$ if there is a $C>0$ such that $a \leq C b$, and $a \sim b$ if $a \lesssim b$ and $b \lesssim a$. We denote the conjugate exponent of $1 \leq r \leq \infty$ by $r^{\prime}$, i.e. $1 / r+1 / r^{\prime}=1$.

\section{2. $S_{p}$-type and $S_{q}$-cotype of operator spaces}

2.1. Definition and basic properties. As an operator space version of absolutely $p$-summing operators G. Pisier introduced completely $p$-summing maps in [21] as follows. 
A linear map $u: E \rightarrow F$ between operator spaces is called completely $p$-summing for $1 \leq p \leq \infty$ if

$$
I_{S_{p}} \otimes u: S_{p} \otimes_{\min } E \rightarrow S_{p}(F)
$$

is a bounded map. We denote by $\pi_{p}^{o}(u)$ the operator norm of $I_{S_{p}} \otimes u$. Similarly we define an operator space version of absolutely $(q, 2)$-summing operators.

A linear map $u: E \rightarrow F$ between operator spaces is called completely $(q, 2)$-summing for $2 \leq q \leq \infty$ if

$$
I_{2, q} \otimes u: S_{2} \otimes \min E \rightarrow S_{q}(F)
$$

is a bounded map, where $I_{2, q}$ is the formal identity from $S_{2}$ into $S_{q}$. We write $\pi_{q, 2}^{o}(u)$ for the operator norm of $I_{2, q} \otimes u$ and $\Pi_{q, 2}^{o}(E, F)$ for the collection of all such operators from $E$ into $F$. Now we define $S_{p}$-type and $S_{q}$-cotype.

Definition 2.1. Let $E$ be an operator space.

(1) $E$ is said to have $S_{p}$-type $(1 \leq p \leq 2)$ if there is a constant $C>0$ such that

$$
\pi_{p^{\prime}, 2}^{o}\left(v^{*}\right) \leq C \cdot \ell^{*}(v)
$$

for every $n \in \mathbb{N}$ and $v: E \rightarrow O H_{n}$.

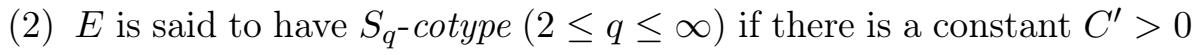
such that

$$
\pi_{q, 2}^{o}(u) \leq C^{\prime} \cdot \ell(u)
$$

for every $n \in \mathbb{N}$ and $u: O H_{n} \rightarrow E$.

We can reformulate $S_{p}$-type and $S_{q}$-cotype by comparing the vectorvalued Schatten class norms of $E$-valued matrices and their gaussian averages. Let $\left\{g_{i j}\right\}$ be a re-indexing of $\left\{g_{i}\right\}$.

Proposition 2.2. Let $E$ be an operator space.

(1) For $n \in \mathbb{N}$ and $1 \leq p \leq 2$ we define $T_{S_{p}, n}(E)$ to be the infimum of the constants $C>0$ satisfying

$$
\left[\int_{\Omega}\left\|\sum_{i, j=1}^{n} g_{i j}(\omega) x_{i j}\right\|^{2} d P(\omega)\right]^{1 / 2} \leq C\left\|\left(x_{i j}\right)\right\|_{S_{p}^{n}(E)} .
$$

$E$ has $S_{p}$-type if and only if

$$
T_{S_{p}}(E)=\sup _{n \geq 1} T_{S_{p}, n}(E)<\infty .
$$

(2) For $n \in \mathbb{N}$ and $2 \leq q \leq \infty$ we define $C_{S_{q}, n}(E)$ to be the infimum of the constants $C^{\prime}>0$ satisfying

$$
\left\|\left(x_{i j}\right)\right\|_{S_{q}^{n}(E)} \leq C^{\prime}\left[\iint_{\Omega}\left\|\sum_{i, j=1}^{n} g_{i j}(\omega) x_{i j}\right\|^{2} d P(\omega)\right]^{1 / 2} .
$$

$E$ has $S_{q}$-cotype if and only if

$$
C_{S_{q}}(E)=\sup _{n \geq 1} C_{S_{q}, n}(E)<\infty .
$$


Proof. (1) E satisfies (2.1) if and only if there is a constant $C>0$ such that

$$
\ell(u) \leq C\left\|\left(u e_{i j}\right)\right\|_{S_{p}^{n}(E)}
$$

for all $n \in \mathbb{N}$ and $u: S_{2}^{n} \rightarrow E$. By trace duality this is equivalent to

$$
\left\|\left(v^{*} e_{i j}\right)\right\|_{S_{p^{\prime}}^{n}\left(E^{*}\right)} \leq C \cdot \ell^{*}(v)
$$

for all $n \in \mathbb{N}$ and $v: E \rightarrow S_{2}^{n}$. Indeed, by Corollary 1.8 of [20] we have

$$
|\operatorname{tr}(v u)|=\left|\sum_{i, j}\left\langle v u e_{i j}, e_{i j}\right\rangle\right|=\left|\sum_{i, j}\left\langle u e_{i j}, v^{*} e_{i j}\right\rangle\right| \leq\left\|\left(u e_{i j}\right)\right\|_{S_{p}^{n}(E)}\left\|\left(v^{*} e_{i j}\right)\right\|_{S_{q}^{n}\left(E^{*}\right)} .
$$

Now we assume that $E$ satisfies (2.1) and consider $v: E \rightarrow O H_{n}$ and $\left(x_{i j}\right) \in M_{m}\left(O H_{n}\right), m \in \mathbb{N}$. If we set $w: S_{2}^{m} \rightarrow O H_{n}, e_{i j} \mapsto x_{i j}$, then by 12.5 of [26] we have

$$
\begin{aligned}
\left\|\left(v^{*} x_{i j}\right)\right\|_{S_{p^{\prime}}^{m}\left(E^{*}\right)} & =\left\|\left(v^{*} w e_{i j}\right)\right\|_{S_{p^{\prime}}^{m}\left(E^{*}\right)} \leq C \cdot \ell^{*}\left(w^{*} v\right) \\
& =C \sup \left\{\left|\operatorname{tr}\left(u w^{*} v\right)\right|: \ell\left(u: S_{2}^{m} \rightarrow E\right) \leq 1\right\} \\
& \leq C \sup \left\{|\operatorname{tr}(\widetilde{u} v)|: \ell\left(\widetilde{u}: O H_{n} \rightarrow E\right) \leq 1\right\}\|w\| \\
& =C \cdot \ell^{*}(v)\|w\|_{\mathrm{cb}}=C \cdot \ell^{*}(v)\left\|\left(x_{i j}\right)\right\|_{S_{2}^{m} \otimes_{\min } O H_{n} .}
\end{aligned}
$$

The converse is straightforward from the above observation and the fact that

$$
\left\|\left(e_{i j}\right)\right\|_{S_{2}^{n} \otimes_{\min } S_{2}^{n}}=\left\|I_{S_{2}^{n}}\right\|_{\mathrm{cb}}=1 .
$$

(2) Suppose $E$ satisfies (2.2) and let $u: O H_{n} \rightarrow E$ and $\left(x_{i j}\right) \in S_{q}^{m}\left(O H_{n}\right)$ for $m \in \mathbb{N}$. If we set $v: S_{2}^{m} \rightarrow O H_{n}, e_{i j} \mapsto x_{i j}$, then $\|v\|=\|v\|_{\mathrm{cb}}=$ $\left\|\left(x_{i j}\right)\right\|_{S_{2}^{m} \otimes_{\min } O H_{n}}$. Now, by (12.5) of [26] we have

$$
\begin{aligned}
\left\|\left(u x_{i j}\right)\right\|_{S_{q}^{m}(E)} & =\left\|\left(u v e_{i j}\right)\right\|_{S_{q}^{m}(E)} \leq C^{\prime}\left\|\sum_{1 \leq i, j \leq m} g_{i j}(\cdot) u v e_{i j}\right\|_{L_{2}(\Omega, E)} \\
& =C^{\prime} \ell(u v) \leq C^{\prime} \ell(u)\|v\|=C^{\prime} \ell(u)\left\|\left(x_{i j}\right)\right\|_{S_{2}^{m} \otimes_{\min } O H_{n}} .
\end{aligned}
$$

The converse direction is straightforward as before.

REMARK 2.3. (1) If we take diagonals of (2.1) and (2.2), then it is trivial that every $S_{p}$-type (resp. $S_{q}$-cotype) space has type $p$ (resp. cotype $q$ ) as a Banach space.

(2) Instead of gaussian systems we can use the Rademacher system $\left\{r_{i}\right\}$ defined by $r_{i}(t)=\operatorname{sign}\left(\sin \left(2^{i} \pi t\right)\right), t \in[0,1]$ and $i=1,2, \ldots$, in the definition to get the Rademacher $S_{p}$-type and $S_{q}$-cotype. It is easy to check that the two notions are equivalent when $1<p \leq 2 \leq q<\infty$. Although we do not know the equivalence for $p=1$ and $q=\infty$ all the calculations in this paper can be transferred to the Rademacher setting with the same arguments.

(3) Unlike the Banach space case, $S_{1}$-type and $S_{\infty}$-cotype are no more trivial, that is, we have examples of operator spaces without $S_{1}$-type and 
$S_{\infty}$-cotype, respectively. We will see examples in Lemma 2.11 and Theorem 2.13 in detail. Moreover, for any operator space $E$ we have

$$
T_{S_{1}, n}(E) \lesssim n^{1 / 2} \quad \text { and } \quad C_{S_{\infty}, n}(E) \lesssim n^{1 / 2} .
$$

Indeed,

$$
\begin{aligned}
\left\|\sum_{i, j=1}^{n} r_{i j} \otimes x_{i j}\right\|_{L_{1}(\Omega, E)} & =\int_{\Omega}\left\|\sum_{i, j=1}^{n} x_{i j} g_{i j}(\omega)\right\| d P(\omega) \\
& \leq \int_{\Omega}\left\|\left(g_{i j}(\omega)\right)\right\|_{S_{\infty}^{n}}\left\|\left(x_{i j}\right)\right\|_{S_{1}^{n}(E)} d P(\omega) \\
& \lesssim n^{1 / 2}\left\|\left(x_{i j}\right)\right\|_{S_{1}^{n}(E)}
\end{aligned}
$$

by Lemma 2.3 of [5] and Proposition 45.1 of [26]. The estimation for $C_{S_{\infty}, n}(E)$ can be obtained by the duality below (Proposition 2.4).

(4) We consider the following transforms:

$$
\mathcal{F}_{\mathcal{G}}: f \mapsto\left(\int_{\Omega} f(t) g_{i j}(\omega) d P(\omega)\right)_{i, j} \quad \text { and } \quad \mathcal{F}_{\mathcal{G}}^{-1}:\left(x_{i j}\right) \mapsto \sum_{i, j} g_{i j}(\omega) x_{i j}
$$

for appropriate $f: \Omega \rightarrow \mathbb{C}$ and $\left(x_{i j}\right) \in M_{\infty}$. Then $E$ has $S_{p}$-type $(1 \leq p \leq 2)$ if and only if

$$
\mathcal{F}_{\mathcal{G}}^{-1} \otimes I_{E}: S_{p}(E) \rightarrow \mathcal{G}_{2}(E)
$$

is bounded, and $E$ has $S_{q}$-cotype $(2 \leq q \leq \infty)$ if and only if

$$
\mathcal{F}_{\mathcal{G}} \otimes I_{E}: \mathcal{G}_{2}(E) \rightarrow S_{q}(E)
$$

is bounded, where $\mathcal{G}_{r}(E)$ is the closed linear span of $\left\{g_{i j}\right\} \otimes E$ in $L_{r}(\Omega, E)$ for $1 \leq r<\infty$. We write $\mathcal{G}_{r}^{n}(E)(n \in \mathbb{N})$ for the closed linear span of $\left\{g_{i j}\right\}_{i, j=1}^{n} \otimes E$ in $L_{r}(\Omega, E)$.

$S_{p}$-type and $S_{q}$-cotype have a partial duality as follows. The proof is the same as in the Banach space case, so we omit it. Note that we can include the cases of $S_{1}$-type and $S_{\infty}$-cotype without any extra effort (see Propositions 11.10 and 13.17 in [2]).

Proposition 2.4. Let $E$ be an operator space, $1 \leq p \leq 2$ and $n \in \mathbb{N}$.

(1) If $E$ has $S_{p^{-}}$-type, then $E^{*}$ has $S_{p^{\prime}}$-cotype with $C_{S_{p^{\prime}}, n}\left(E^{*}\right) \leq T_{S_{p}, n}(E)$.

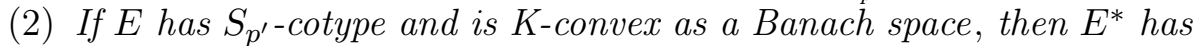

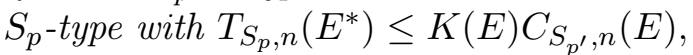

where $K(E)$ is the $K$-convexity constant of $E$ defined by the operator norm of the $E$-valued gaussian projection from $L_{2}(\Omega, E)$ onto $\mathcal{G}_{2}(E)$, given by

$$
f \mapsto \sum_{i, j}\left(\int_{\Omega} f(t) g_{i j}(\omega) d P(\omega)\right) g_{i j}
$$


2.2. Relation to other concepts. Now we check that the $S_{2}$-cotype of this paper coincides with the $O H$-cotype 2 of [18]. This can be achieved by the following trace duality for the $\pi_{2}^{o}$-norm. It is well-known to experts, but we include the proof since we could not find a reference.

Lemma 2.5. Let $E$ and $F$ be operator spaces and $E$ be finite-dimensional. Then for $v: F \rightarrow E$ we have

$$
\left(\pi_{2}^{o}\right)^{*}(v):=\sup \left\{|\operatorname{tr}(v u)| \mid \pi_{2}^{o}(u: E \rightarrow F) \leq 1\right\}=\pi_{2}^{o}(v) .
$$

Proof. Let $u: E \rightarrow F$ and $v: F \rightarrow E$. By Proposition 6.1 of [21], we have factorizations

$$
u: E \stackrel{V_{1}}{\rightarrow} O H(I) \stackrel{T_{1}}{\rightarrow} F \quad \text { and } \quad v: F \stackrel{V_{2}}{\rightarrow} O H(J) \stackrel{T_{2}}{\rightarrow} E
$$

for some index sets $I$ and $J$ with

$$
\pi_{2}^{o}\left(V_{1}\right), \quad \pi_{2}^{o}\left(V_{2}\right) \leq 1, \quad\left\|T_{1}\right\|_{\mathrm{cb}} \leq \pi_{2}^{o}(u), \quad\left\|T_{2}\right\|_{\mathrm{cb}} \leq \pi_{2}^{o}(v) .
$$

Then, by Proposition 6.3 of [21] we have

$$
\begin{aligned}
|\operatorname{tr}(v u)| & =\left|\operatorname{tr}\left(T_{2} V_{2} T_{1} V_{1}\right)\right|=\left|\operatorname{tr}\left(V_{1} T_{2} V_{2} T_{1}\right)\right| \\
& \leq\left\|V_{2} T_{1}\right\|_{\mathrm{HS}}\left\|V_{1} T_{2}\right\|_{\mathrm{HS}}=\pi_{2}^{o}\left(V_{2} T_{1}\right) \pi_{2}^{o}\left(V_{1} T_{2}\right) \\
& \leq \pi_{2}^{o}\left(V_{2}\right)\left\|T_{1}\right\|_{\mathrm{cb}} \pi_{2}^{o}\left(V_{1}\right)\left\|T_{2}\right\|_{\mathrm{cb}} \leq \pi_{2}^{o}(v) \pi_{2}^{o}(u),
\end{aligned}
$$

where $\|\cdot\|_{\text {HS }}$ is the Hilbert-Schmidt norm.

Thus, we get $\left(\pi_{2}^{o}\right)^{*}(v) \leq \pi_{2}^{o}(v)$.

For the opposite inequality we consider any $\varepsilon>0$ and choose $\left(x_{i j}\right) \in$ $S_{2}^{n} \otimes_{\min } F$ with

$$
\left\|\left(x_{i j}\right)\right\|_{S_{2}^{n} \otimes_{\min } F}=1 \quad \text { and } \quad\left\|\left(v x_{i j}\right)\right\|_{S_{2}^{n}(E)} \geq(1-\varepsilon) \pi_{2}^{o}(v) .
$$

Then there is $\left(y_{i j}^{*}\right) \in S_{2}^{n}\left(E^{*}\right)$ such that

$$
\left\|\left(y_{i j}^{*}\right)\right\|_{S_{2}^{n}\left(E^{*}\right)}=1 \quad \text { and }\left\|\left(v x_{i j}\right)\right\|_{S_{2}^{n}(E)}=\left|\left\langle\left(v x_{i j}\right),\left(y_{i j}^{*}\right)\right\rangle\right| \text {. }
$$

Now we set $A: E \rightarrow S_{2}^{n}, x \mapsto\left(y_{i j}^{*} x\right)_{i, j}$, and $B: S_{2}^{n} \rightarrow F, e_{i j} \mapsto x_{i j}$. Then we get

$$
\pi_{2}^{o}(B A) \leq\|B\|_{\mathrm{cb}} \pi_{2}^{o}(A) \leq\left\|\left(y_{i j}^{*}\right)\right\|_{S_{2}^{n}(E)}\left\|\left(x_{i j}\right)\right\|_{S_{2}^{n} \otimes_{\min } F} \leq 1
$$

by Lemma 5.14 of [21]. Thus, we have

$$
\begin{aligned}
\left(\pi_{2}^{o}\right)^{*}(v) & \geq|\operatorname{tr}(v B A)|=|\operatorname{tr}(A v B)|=\sum_{i, j=1}^{n}\left|\left\langle A v B e_{i j}, e_{i j}\right\rangle\right| \\
& =\left|\left\langle\left(v x_{i j}\right),\left(y_{i j}^{*}\right)\right\rangle\right| \geq(1-\varepsilon) \pi_{2}^{o}(v),
\end{aligned}
$$

which gives us the opposite inequality.

Corollary 2.6. The $S_{2}$-cotype coincides with the OH-cotype 2 of [18].

Proof. By Proposition 6.2 of [21], we have $\pi_{2}^{o}(v)=\pi_{2, \mathrm{oh}}(v)$ for any $v$ : $E \rightarrow O H_{n}$. Thus, we get the desired conclusion by Lemma 2.5 and trace duality. 
We end this section by providing a partial relationship between $S_{2}$-type and $S_{2}$-cotype and notions in [6].

Let $(\Omega, P)$ be a probability space and $\left(\Sigma, d_{\Sigma}\right)$ be a pair of an index set $\Sigma$ and a collection of natural numbers indexed by $\Sigma, d_{\Sigma}=\left\{d_{\sigma} \in \mathbb{N}: \sigma \in \Sigma\right\}$. The quantized gaussian system $\mathcal{G}_{\Sigma}$ with parameter $\left(\Sigma, d_{\Sigma}\right)$ is the collection of random matrices $g^{\sigma}=d_{\sigma}^{-1 / 2}\left(g_{i j}^{\sigma}\right): \Omega \rightarrow M_{d_{\sigma}}$ indexed by $\Sigma$, where $g_{i j}^{\sigma}$ 's are i.i.d. gaussian random variables. We consider the following transforms:

$\mathcal{F}_{\mathcal{G}_{\Sigma}}(f)(\sigma)=\int_{\Omega} f(\omega) g^{\sigma}(\omega)^{*} d P(\omega) \quad$ and $\quad \mathcal{F}_{\mathcal{G}_{\Sigma}}^{-1}(A)(\omega)=\sum_{\sigma \in \Sigma} d_{\sigma} \operatorname{tr}\left(A^{\sigma} g^{\sigma}(\omega)\right)$ for appropriate $f: \Omega \rightarrow \mathbb{C}$ and $A \in \prod_{\sigma \in \Sigma} M_{d_{\sigma}}$.

For $1 \leq p \leq 2,2 \leq q \leq \infty, 1 / p+1 / p^{\prime}=1$ and $1 / q+1 / q^{\prime}=1$ we say that an operator space $E$ has Banach $\mathcal{G}_{\Sigma^{-}}$-type $p$ if

$$
\sup _{\text {finite } \Gamma \subseteq \Sigma}\left\|\mathcal{F}_{\mathcal{G}_{\Sigma}}^{-1} \otimes I_{E}\right\|_{\mathcal{L}_{p}(\Gamma, E) \rightarrow L_{p^{\prime}}(\Omega, E)}<\infty
$$

and that $E$ has Banach $\mathcal{G}_{\Sigma}$-cotype $q$ if

$$
\sup _{\text {finite } \Gamma \subseteq \Sigma}\left\|\mathcal{F}_{\mathcal{G}_{\Sigma}} \otimes I_{E}\right\|_{L_{q^{\prime}}^{\Gamma}(\Omega, E) \rightarrow \mathcal{L}_{q}(\Gamma, E)}<\infty
$$

where $L_{q^{\prime}}^{\Gamma}(\Omega, E)$ is the closed linear span of $\left\{g_{i j}^{\sigma}: \sigma \in \Gamma\right\} \otimes E$ in $L_{q^{\prime}}(\Omega, E)$, $\mathcal{L}_{r}(\Gamma, E)=\left\{A \in \prod_{\sigma \in \Gamma} M_{d_{\sigma}} \otimes E:\|A\|_{\mathcal{L}_{r}(\Gamma, E)}=\left(\sum_{\sigma \in \Gamma} d_{\sigma}\left\|A^{\sigma}\right\|_{S_{r}^{d \sigma}(E)}^{r}\right)^{1 / r}<\infty\right\}$ for $1 \leq r<\infty$ and

$$
\mathcal{L}_{\infty}(\Gamma, E)=\left\{A \in \prod_{\sigma \in \Gamma} M_{d_{\sigma}} \otimes E:\|A\|_{\mathcal{L}_{\infty}(\Gamma, E)}=\sup _{\sigma \in \Gamma}\left\|A^{\sigma}\right\|_{S_{\infty}^{d_{\sigma}}(E)}<\infty\right\} .
$$

For the details and the natural operator space structure on $\mathcal{L}_{r}(\Gamma, E)$, see $[5,21]$.

Proposition 2.7. Let $E$ be an operator space and $\mathcal{G}_{\Sigma}$ be the quantized gaussian system with parameter $\left(\Sigma, d_{\Sigma}\right)$. Suppose that $d_{\Sigma}$ is unbounded. Then $E$ has gaussian $S_{2}$-type if and only if it has Banach $\mathcal{G}_{\Sigma}$-type 2 , and $E$ has gaussian $S_{2}$-cotype if and only if it has Banach $\mathcal{G}_{\Sigma}$-cotype 2 .

Proof. Let $\Gamma$ be a finite subset of $\Sigma$ and $A\left(=\left(A^{\sigma}\right)\right) \in \prod_{\sigma \in \Gamma} M_{d_{\sigma}} \otimes E$. If we set

$$
B=\bigoplus_{\sigma \in \Gamma} \sqrt{d_{\sigma}} A^{\sigma} \in S_{\infty}^{n}(E)
$$

for $n=\sum_{\sigma \in \Gamma} d_{\sigma}$, then we get

$$
\mathcal{F}_{\mathcal{G}_{\Sigma}}^{-1}(A)(\omega)=\sum_{\sigma \in \Gamma} d_{\sigma} \operatorname{tr}\left(A^{\sigma} g^{\sigma}(\omega)\right)=\sum_{\sigma \in \Gamma} \sqrt{d_{\sigma}} \operatorname{tr}\left(A^{\sigma}\left(g_{i j}^{\sigma}(\omega)\right)\right)=\mathcal{F}_{\mathcal{G}}^{-1}(B)(\omega)
$$


and

$$
\|A\|_{\mathcal{L}_{2}(\Gamma, E)}=\left[\sum_{\sigma \in \Gamma} d_{\sigma}\left\|A^{\sigma}\right\|_{S_{2}^{\sigma}(E)}^{2}\right]^{1 / 2}=\|B\|_{S_{2}^{n}(E)} .
$$

Conversely, for any $B \in S_{\infty}^{n}(E)$ we choose $\sigma_{0} \in \Sigma$ with $d_{\sigma_{0}}>n$ and define $A\left(=\left(A^{\sigma}\right)\right) \in \prod_{\sigma \in \Sigma} M_{d_{\sigma}} \otimes E$ by $A^{\sigma_{0}}=d_{\sigma_{0}}^{-1 / 2} B \oplus 0$ and $A^{\sigma}=0$ elsewhere. Then we also get $\mathcal{F}_{\mathcal{G}_{\Sigma}}^{-1}(A)=\mathcal{F}_{\mathcal{G}}^{-1}(B)$ and $\|A\|_{\mathcal{L}_{2}(\Sigma, E)}=\|B\|_{S_{2}^{n}(E)}$. Thus, we get the desired result.

2.3. $S_{p}$-type and $S_{q}$-cotype of $R[p]$ and $C[p]$. In the case of Hilbertian spaces the gaussian average of a vector-valued matrix is easy to calculate so that we can determine $S_{p}$-type and $S_{q}$-cotype in some concrete cases.

Theorem 2.8. Let $1 \leq p \leq \infty$ and $1 / p+1 / p^{\prime}=1$. Then both $R[p]$ and $C[p]$ have $S_{\min \left\{p, p^{\prime}\right\}}$-type and $S_{\max \left\{p, p^{\prime}\right\} \text {-cotype, and neither of them has }}$

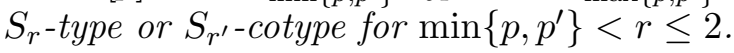

Proof. Note that $R$ and $R_{n}$ are isometric to $O H$ and $O H_{n}$, respectively. Thus,

$R$ has $S_{q}$-cotype $\Leftrightarrow \mathcal{F}_{\mathcal{R}} \otimes I_{R}: \mathcal{G}_{2}(R) \rightarrow S_{q}(R)$ is bounded

$\Leftrightarrow \mathcal{F}_{\mathcal{R}} \otimes \mathrm{id}: \mathcal{G}_{2}(O H) \rightarrow S_{q}(R)$ is bounded

$\Leftrightarrow I_{2, q} \otimes \mathrm{id}: S_{2}(O H) \rightarrow S_{q}(R)$ is bounded

$\Leftrightarrow I_{2, q}^{n} \otimes \mathrm{id}_{n}: S_{2}^{n}\left(O H_{n}\right) \rightarrow S_{q}^{n}\left(R_{n}\right)$ is uniformly bounded

for all $n \in \mathbb{N}$, where id, $\operatorname{id}_{n}, I_{2, q}$ and $I_{2, q}^{n}$ are the corresponding formal identities.

First, we consider the case $q=\infty$. For $\left(x_{i j}\right) \in S_{\infty}^{n}\left(R_{n}\right), x_{i j}=\sum_{k=1}^{n} x_{i j}^{k} e_{1 k}$, we have

$$
\left\|\left(x_{i j}\right)\right\|_{S_{2}^{n}\left(O H_{n}\right)}=\left(\sum_{i, j, k=1}^{n}\left|x_{i j}^{k}\right|^{2}\right)^{1 / 2},
$$

and by considering $\left(x_{i j}\right)$ as an $n^{2} \times n^{2}$-matrix,

$$
\left\|\left(x_{i j}\right)\right\|_{S_{\infty}^{n}\left(R_{n}\right)}=\sup \left\{\left.\left[\sum_{i=1}^{n}\left(\sum_{j, k=1}^{n} x_{i j}^{k} a_{j k}\right)^{2}\right]^{1 / 2}\left|\sum_{j, k=1}^{n}\right| a_{j k}\right|^{2}=1\right\} .
$$

However, we have

$$
\begin{aligned}
{\left[\sum_{i=1}^{n}\left(\sum_{j, k=1}^{n} x_{i j}^{k} a_{j k}\right)^{2}\right]^{1 / 2} } & \leq\left[\sum_{i=1}^{n}\left(\sum_{j, k=1}^{n}\left|x_{i j}^{k}\right|^{2}\right)\left(\sum_{j, k=1}^{n}\left|a_{j k}\right|^{2}\right)\right]^{1 / 2} \\
& =\left(\sum_{i, j, k=1}^{n}\left|x_{i j}^{k}\right|^{2}\right)^{1 / 2}\left(\sum_{j, k=1}^{n}\left|a_{j k}\right|^{2}\right)^{1 / 2}
\end{aligned}
$$

and consequently

$$
\left\|\left(x_{i j}\right)\right\|_{S_{\infty}^{n}\left(R_{n}\right)} \leq\left\|\left(x_{i j}\right)\right\|_{S_{2}^{n}\left(O H_{n}\right)} .
$$


Thus, $R$ has $S_{\infty}$-cotype with $C_{S_{\infty}}(R)=1$, and we can similarly show that $C$ has $S_{\infty}$-cotype with $C_{S_{\infty}}(C)=1$. Since $R$ and $C$ are $K$-convex as Banach spaces, they have $S_{1}$-type by duality (Proposition 2.4). Since $R[2]$ (resp. $C[2]$ ) is completely isometric to $O H$, it has $S_{2}$-type and $S_{2}$-cotype. Thus by complex interpolation, both $R[p]$ and $C[p]$ have $S_{\min \left\{p, p^{\prime}\right\}^{-t y p e}}$ and $S_{\max \left\{p, p^{\prime}\right\}^{-}}$ cotype.

Now suppose $2 \leq q<p \leq \infty$ and consider $S_{q}(R[p])$. By Theorem 1.1 of [21],

$$
S_{q}(R[p]) \cong C[q] \otimes_{h} R[p] \otimes_{h} R[q]
$$

completely isometrically under the mapping

$$
e_{i j} \otimes x \mapsto e_{i 1} \otimes x \otimes e_{1 j},
$$

where $\otimes_{h}$ is the Haagerup tensor product. Note that by the commutation property of the Haagerup tensor product with respect to complex interpolation we have the following completely isometric isomorphisms:

$$
\begin{aligned}
R[p] \otimes_{h} R[q] & \cong\left[R[p] \otimes_{h} R, R[p] \otimes_{h} C\right]_{1 / q} \\
& \cong\left[\left[R \otimes_{h} R, C \otimes_{h} R\right]_{1 / p},\left[R \otimes_{h} C, C \otimes_{h} C\right]_{1 / p}\right]_{1 / q} .
\end{aligned}
$$

Since $C \otimes_{h} R$ (resp. $R \otimes_{h} C$ ) is completely isometric to $S_{\infty}$ (resp. $S_{1}$ ), and $R \otimes_{h} R$ and $C \otimes_{h} C$ are both isometric to $S_{2}$, we get a subspace

$$
F\left(\cong R[p] \otimes_{h} R[q]\right) \text { of } S_{q}(R[p]) \text { isometric to } S_{r}
$$

under the mapping $e_{1 j} \otimes e_{1 i} \mapsto e_{i j}$, where $r=2 p q /(p q+p-q)$. However, we have

$$
\left(I_{2, q} \otimes \mathrm{id}\right)^{-1}\left(e_{1 j} \otimes e_{1 i}\right)=e_{1 j} \otimes e_{i} \in S_{2}(O H),
$$

so that

$$
G=\left(I_{2, q} \otimes \mathrm{id}\right)^{-1}(F) \cong S_{2}
$$

isometrically.

Consequently, $I_{2, q} \otimes$ id cannot be bounded since $r<2$ and $\left(I_{2, q} \otimes\right.$ id) $\left.\right|_{G}$ is nothing but the formal identity $I_{2, r}: S_{2} \rightarrow S_{r}$, which means $R[p]$ does not have $S_{q}$-cotype. We can show that $C[p]$ does not have $S_{q}$-cotype similarly, and the type cases are obtained by duality (Proposition 2.4 ). Since $R\left[p^{\prime}\right] \cong C[p]$ we get the desired result for all $1 \leq p \leq \infty$.

2.4. $S_{p}$-type and $S_{q}$-cotype of $L_{p}$ spaces. In this section we will compute $S_{p}$-type and $S_{q}$-cotype of $L_{p}$ spaces. Unfortunately, we do not have good behavior as in the Banach space case generally. We only have the same results as in the Banach space case for $L_{p}$ spaces $(1 \leq p<\infty)$ with respect to Type I von Neumann algebras of bounded degree. When $p=\infty$, we have very bad behavior even in the commutative cases.

Theorem 2.9. Let $(\mathcal{M}, \mu)$ be a $\sigma$-finite measure space, $1 \leq p<\infty$ and $n \in \mathbb{N}$. 
(1) $L_{p}\left(\mu, S_{p}^{n}\right)$ has $S_{r}$-type and $S_{r^{\prime}}$-cotype for $r=\min \{p, 2\}$ and $1 / r+$ $1 / r^{\prime}=1$. If $L_{p}(\mu)$ is infinite-dimensional, then it does not have $S_{r^{-}}$ type nor $S_{r^{\prime}}$-cotype for any $\min \{p, 2\}<r \leq 2$.

(2) $L_{\infty}\left(\mu, S_{\infty}^{n}\right)$ has $S_{\infty}$-cotype. If $L_{\infty}(\mu)$ is infinite-dimensional, then it does not have $S_{1}$-type nor $S_{q}$-cotype for any $q<\infty$.

Proof. Note that the $p=2$ cases are trivial. First, we consider the case $1 \leq p \leq 2$. For $\left(x_{i j}\right) \in S_{2}\left(L_{1}\left(\mu, S_{1}^{n}\right)\right)$, we have

$$
\begin{aligned}
\left\|\sum_{i, j} g_{i j} \otimes x_{i j}\right\|_{L_{2}\left(\Omega, L_{1}\left(\mu, S_{1}^{n}\right)\right)} & \geq\left\|\sum_{i, j} g_{i j} \otimes x_{i j}\right\|_{L_{1}\left(\Omega, L_{1}\left(\mu, S_{1}^{n}\right)\right)} \\
& =\int_{\mathcal{M}}\left\|\sum_{i, j} x_{i j}(s) \otimes g_{i j}\right\|_{S_{1}^{n}\left(L_{1}(\Omega)\right)} d \mu(s) .
\end{aligned}
$$

Since $\mathcal{G}_{1}$ and $S_{2}$ are isomorphic as Banach spaces, $S_{1}^{n}\left(\mathcal{G}_{1}\right)$ and $S_{1}^{n}\left(S_{2}\right)$ are isomorphic allowing constants depending on $n$. Indeed, we have $S_{1}^{n}\left(\mathcal{G}_{1}\right)^{*}=$ $C B\left(\mathcal{G}_{1}, S_{\infty}^{n}\right) \cong B\left(\mathcal{G}_{1}, S_{\infty}^{n}\right) \cong B\left(S_{2}, S_{\infty}^{n}\right) \cong C B\left(S_{2}, S_{\infty}^{n}\right)=S_{1}^{n}\left(S_{2}\right)^{*}$ isomorphically. Thus, by Corollary 1.10 of [21] we have

$$
\begin{aligned}
\left\|\sum_{i, j} g_{i j} \otimes x_{i j}\right\|_{L_{2}\left(\Omega, L_{1}\left(\mu, S_{1}^{n}\right)\right)} & \gtrsim \int_{\mathcal{M}}\left\|\sum_{i, j} x_{i j}(s) \otimes e_{i j}\right\|_{S_{1}^{n}\left(S_{2}\right)} d \mu(s) \\
& \geq\left\|\left(x_{i j}\right)\right\|_{S_{2}\left(L_{1}\left(\mu, S_{1}^{n}\right)\right) .}
\end{aligned}
$$

Thus, $L_{1}\left(\mu, S_{1}^{n}\right)$ has $S_{2}$-cotype and by complex interpolation with $L_{2}\left(\mu, S_{2}^{n}\right)$ we find that $L_{p}\left(\mu, S_{p}^{n}\right)(1 \leq p \leq 2)$ has $S_{2}$-cotype.

Since $S_{1} \stackrel{I_{1,2}}{\longrightarrow} S_{2} \stackrel{\mathcal{F}_{\mathcal{R}}^{-1}}{\longrightarrow} \mathcal{G}_{2} \subseteq L_{2}(\Omega)$ is a contraction, where $I_{1,2}$ is the corresponding formal identity, so is

$$
\mathcal{F}_{\mathcal{R}}^{-1} \otimes I_{L_{1}\left(\mu, S_{1}^{n}\right)}: S_{1} \otimes_{\gamma} L_{1}\left(\mu, S_{1}^{n}\right) \rightarrow L_{2}(\Omega) \otimes_{\gamma} L_{1}\left(\mu, S_{1}^{n}\right),
$$

where $\otimes_{\gamma}$ is the projective tensor product in the category of Banach spaces. Note that $L_{2}(\Omega) \otimes_{\gamma} E \hookrightarrow L_{2}(\Omega, E)$ contractively by the canonical embedding, and

$$
S_{1} \otimes_{\gamma} L_{1}\left(\mu, S_{1}^{n}\right) \cong S_{1}\left(L_{1}\left(\mu, S_{1}^{n}\right)\right)
$$

isomorphically. Indeed, we have

$$
\begin{aligned}
\left(S_{1} \otimes_{\gamma} L_{1}\left(\mu, S_{1}^{n}\right)\right)^{*} & =B\left(S_{1}\left(L_{1}(\mu)\right), S_{\infty}^{n}\right) \cong C B\left(S_{1}\left(L_{1}(\mu)\right), S_{\infty}^{n}\right) \\
& =S_{1}\left(L_{1}\left(\mu, S_{1}^{n}\right)\right)^{*}
\end{aligned}
$$

isomorphically (allowing constants depending on $n$ ). Thus, we have a bounded map

$$
\mathcal{F}_{\mathcal{R}}^{-1} \otimes I_{L_{1}\left(\mu, S_{1}^{n}\right)}: S_{1}\left(L_{1}\left(\mu, S_{1}^{n}\right)\right) \rightarrow L_{2}\left(\Omega, L_{1}\left(\mu, S_{1}^{n}\right)\right),
$$

which implies that $L_{1}\left(\mu, S_{1}^{n}\right)$ has $S_{1}$-type. By complex interpolation with respect to $L_{2}\left(\mu, S_{2}^{n}\right)$ we see that $L_{p}\left(\mu, S_{p}^{n}\right)(1 \leq p \leq 2)$ has $S_{p}$-type. 
Now we consider the case $2 \leq p<\infty$. We can show that $L_{p}\left(\mu, S_{p}^{n}\right)$ has $S_{2}$-type by a direct calculation as above. Since $\mathcal{G}_{2} \stackrel{\mathcal{F}_{\mathcal{R}}}{\longrightarrow} S_{2} \stackrel{I_{2, \infty}}{\longrightarrow} S_{\infty}$ is a contraction, where $I_{2, \infty}$ is the corresponding formal identity, so is

$$
\mathcal{F}_{\mathcal{R}} \otimes I_{L_{\infty}\left(\mu, S_{\infty}^{n}\right)}: \mathcal{G}_{2} \otimes_{\lambda} L_{\infty}\left(\mu, S_{\infty}^{n}\right) \rightarrow S_{\infty} \otimes_{\lambda} L_{\infty}\left(\mu, S_{\infty}^{n}\right),
$$

where $\otimes_{\lambda}$ is the injective tensor product in the category of Banach spaces. Note that we have the contraction

$$
\mathcal{G}_{2}(E) \subseteq L_{2}(\Omega, E) \hookrightarrow L_{2}(\Omega) \otimes_{\lambda} E \stackrel{P_{2} \otimes I_{E}}{\longrightarrow} \mathcal{G}_{2} \otimes_{\lambda} E,
$$

for $E=L_{\infty}\left(\mu, S_{\infty}^{n}\right)$ and $P_{2}$ is the gaussian projection from $L_{2}(\Omega)$ onto $\mathcal{G}_{2}$. Since

$$
\begin{aligned}
B\left(S_{1}\left(L_{1}(\mu)\right), S_{\infty}^{n}\right) \supseteq S_{\infty} \otimes_{\lambda} & L_{\infty}\left(\mu, S_{\infty}^{n}\right) \\
& \cong S_{\infty}\left(L_{\infty}\left(\mu, S_{\infty}^{n}\right)\right) \subseteq C B\left(S_{1}\left(L_{1}(\mu)\right), S_{\infty}^{n}\right)
\end{aligned}
$$

isomorphically, we have a bounded map

$$
\mathcal{F}_{\mathcal{R}} \otimes I_{L_{\infty}\left(\mu, S_{\infty}^{n}\right)}: \mathcal{G}_{2}\left(L_{\infty}\left(\mu, S_{\infty}^{n}\right)\right) \rightarrow S_{\infty}\left(L_{\infty}\left(\mu, S_{\infty}^{n}\right)\right),
$$

which implies $L_{\infty}\left(\mu, S_{\infty}^{n}\right)$ has $S_{\infty}$-cotype. By complex interpolation with respect to $L_{2}\left(\mu, S_{2}^{n}\right)$ we conclude that $L_{p}\left(\mu, S_{p}^{n}\right)(2 \leq p \leq \infty)$ has $S_{p}$-cotype.

The other statements concerning best $S_{p}$-type and $S_{q}$-cotype follow by Remark 2.3 and the Banach space case, except the fact that an infinitedimensional $L_{\infty}(\mu)$ does not have $S_{1}$-type. For simplicity we just consider the case of $c_{0}$, the space of null sequences. Note that by the dominance of the gaussian average over the Rademacher average (for example, (4.2) of [26]) we have

$$
\begin{aligned}
T_{1}^{o, n}\left(c_{0}\right) & =\left\|S_{1}^{n}\left(c_{0}\right) \rightarrow L_{2}\left(\Omega, c_{0}\right),\left(x_{i j}\right) \mapsto \sum_{i, j=1}^{n} g_{i j} \otimes x_{i j}\right\| \\
& \gtrsim\left\|S_{1}^{n}\left(c_{0}\right) \rightarrow L_{2}\left([0,1], c_{0}\right),\left(x_{i j}\right) \mapsto \sum_{i, j=1}^{n} r_{i j} \otimes x_{i j}\right\| \\
& =\left\|L_{2}\left([0,1], \ell_{1}\right) \rightarrow S_{\infty}^{n}\left(\ell_{1}\right), f \mapsto\left(\left\langle r_{i j}, f\right\rangle\right)_{i, j}\right\|,
\end{aligned}
$$

where $\left\{r_{i j}\right\}$ is a reindexing of the classical Rademacher system $\left\{r_{i}\right\}$.

Set $f(t)=\prod_{i, j=1}^{n}\left(1+r_{i j}(t) r_{i j}\right) \in L_{2}\left([0,1], L_{1}[0,1]\right)$. Then

$$
\|f(t)\|_{L_{1}[0,1]}=\int_{0}^{1}\left|\prod_{i, j=1}^{n}\left(1+r_{i j}(t) r_{i j}(s)\right)\right| d s=\int_{0}^{1} \prod_{i, j=1}^{n}\left(1+r_{i j}(t) r_{i j}(s)\right) d s=1
$$

for all $t \in[0,1]$, and consequently

$$
\|f\|_{L_{2}\left([0,1], L_{1}[0,1]\right)}=1 .
$$


On the other hand, we have $\left\langle r_{i j}, f\right\rangle=r_{i j}$, so that

$$
\begin{aligned}
\left\|\left(\left\langle r_{i j}, f\right\rangle\right)_{i, j}\right\|_{S_{\infty}^{n}\left(L_{1}[0,1]\right)} & \left\|\sum_{i, j=1}^{n} e_{i j} \otimes r_{i j}\right\|_{S_{\infty}^{n}\left(L_{1}[0,1]\right)} \sim\left\|\sum_{i, j=1}^{n} e_{i j} \otimes e_{i j}\right\|_{S_{\infty}^{n}\left(R_{n^{2}}+C_{n^{2}}\right)} \\
& =\left\|R_{n^{2}} \cap C_{n^{2}} \rightarrow S_{\infty}^{n}, \delta_{i j} \mapsto e_{i j}\right\|_{\mathrm{cb}} \\
& \geq \frac{\left\|\sum_{i, j=1}^{n} e_{i j} \otimes e_{i j}\right\|_{S_{\infty}^{n}\left(S_{\infty}^{n}\right)}}{\max \left\{\left\|\sum_{i, j=1}^{n} e_{i j} e_{i j}^{*}\right\|_{\min }^{1 / 2},\left\|\sum_{i, j=1}^{n} e_{i j}^{*} e_{i j}\right\|_{\min }^{1 / 2}\right\}}=n^{1 / 2} .
\end{aligned}
$$

Since $\operatorname{span}\left\{r_{A}=\prod_{(i, j) \in A} r_{i j} \mid A \subseteq\{(i, j) \mid 1 \leq i, j \leq n\}\right\}$ in $L_{1}[0,1]$ is completely isometric to $\ell_{1}^{2^{n^{2}}}$ we get

$$
T_{1}^{o, n}\left(c_{0}\right) \gtrsim n^{1 / 2}
$$

the desired result.

REMARK 2.10. We do not need $\sigma$-finiteness of $\mu$ to prove that $L_{1}\left(\mu, S_{1}^{n}\right)$ has $S_{1}$-type and $L_{\infty}\left(\mu, S_{\infty}^{n}\right)$ has $S_{\infty}$-cotype in the above theorem, and we can similarly show that every maximal operator space has $S_{1}$-type and every minimal operator space has $S_{\infty}$-cotype.

Now, we consider $S_{p}$-type and $S_{q}$-cotype of infinite-dimensional Schatten classes. Unfortunately we have not been able to determine the best $S_{p}$-type and $S_{q}$-cotype of those spaces, but the following estimate shows that they are quite different from type and cotype as Banach spaces.

Lemma 2.11. Let $1 \leq p \leq 2$. Then

$$
T_{S_{p}, n}\left(S_{p}\right) \sim C_{S_{p^{\prime}}, n}\left(S_{p^{\prime}}\right) \sim n^{1 / p-1 / 2} .
$$

Proof. First, we consider type constants. By Theorem 9.8.5 of [22] we have

$$
\begin{aligned}
T_{S_{p}, n}\left(S_{p}\right) & \sim\left\|\mathcal{F}_{\mathcal{R}}^{-1} \otimes I_{S_{p}}: S_{p}^{n}\left(S_{p}\right) \rightarrow \mathcal{G}_{p}^{n}\left(S_{p}\right)\right\|=\left\|S_{p}^{n} \rightarrow \mathcal{G}_{p}^{n}, e_{i j} \mapsto r_{i j}\right\|_{\mathrm{cb}} \\
& \sim\left\|R_{n^{2}}\left[p^{\prime}\right] \cap C_{n^{2}}\left[p^{\prime}\right] \rightarrow S_{p^{\prime}}^{n}, e_{i j} \mapsto e_{i j}\right\|_{\mathrm{cb}} \\
& \leq\left\|R_{n^{2}}\left[p^{\prime}\right] \rightarrow S_{p^{\prime}}^{n}, e_{i j} \mapsto e_{i j}\right\|_{\mathrm{cb}} \\
& =\left\|R_{n}\left[p^{\prime}\right] \otimes_{h} R_{n}\left[p^{\prime}\right] \rightarrow C_{n}\left[p^{\prime}\right] \otimes_{h} R_{n}\left[p^{\prime}\right], e_{1 i} \otimes e_{1 j} \mapsto e_{i 1} \otimes e_{1 j}\right\|_{\mathrm{cb}} \\
& \leq\left\|R_{n}\left[p^{\prime}\right] \rightarrow C_{n}\left[p^{\prime}\right], e_{1 i} \mapsto e_{i 1}\right\|_{\mathrm{cb}} .
\end{aligned}
$$

By complex interpolation for $\theta / 2+(1-\theta) / \infty=1 / p^{\prime}$ we get

$$
\begin{aligned}
& \left\|R_{n}\left[p^{\prime}\right] \rightarrow C_{n}\left[p^{\prime}\right], e_{1 i} \mapsto e_{i 1}\right\|_{\mathrm{cb}} \\
& \quad \leq\left\|R_{2}^{n} \rightarrow C_{2}^{n}, e_{1 i} \mapsto e_{i 1}\right\|_{\mathrm{cb}}^{\theta}\left\|R_{\infty}^{n} \rightarrow C_{\infty}^{n}, e_{1 i} \mapsto e_{i 1}\right\|_{\mathrm{cb}}^{1-\theta}=n^{1 / p-1 / 2} .
\end{aligned}
$$


For the upper bound we consider

$$
\begin{aligned}
& \left\|R_{n^{2}}\left[p^{\prime}\right] \cap C_{n^{2}}\left[p^{\prime}\right] \rightarrow S_{p^{\prime}}^{n}, e_{i j} \mapsto e_{i j}\right\|_{\mathrm{cb}} \\
& \quad \geq \frac{\left\|\sum_{i, j=1}^{n} e_{i j} \otimes e_{i j}\right\|_{S_{p^{\prime}}^{n}\left(S_{p^{\prime}}^{n}\right)}}{\max \left\{\left\|\left(\sum_{i, j=1}^{n} e_{i j}^{*} e_{i j}\right)^{1 / 2}\right\|_{S_{p^{\prime}}^{n}}\left\|\left(\sum_{i, j=1}^{n} e_{i j} e_{i j}^{*}\right)^{1 / 2}\right\|_{\left.S_{p^{\prime}}^{n}\right\}}\right.}=n^{1 / p-1 / 2} .
\end{aligned}
$$

Now we consider cotype constants. For $1<p \leq 2$ we have

$$
\begin{aligned}
C_{S_{p^{\prime}}, n}\left(S_{p^{\prime}}\right) & \sim\left\|\mathcal{F}_{\mathcal{R}} \otimes I_{S_{p}}: \mathcal{G}_{p^{\prime}}^{n}\left(S_{p^{\prime}}\right) \rightarrow S_{p^{\prime}}^{n}\left(S_{p^{\prime}}\right)\right\| \\
& \sim\left\|R_{n^{2}}\left[p^{\prime}\right] \cap C_{n^{2}}\left[p^{\prime}\right] \rightarrow S_{p^{\prime}}^{n}, e_{i j} \mapsto e_{i j}\right\|_{\mathrm{cb}}=n^{1 / p-1 / 2} .
\end{aligned}
$$

For $p=1$ we have, by Proposition 45.1 of [26],

$$
\begin{aligned}
C_{\infty}^{o, n}\left(S_{\infty}\right) & \sim\left\|\mathcal{F}_{\mathcal{R}} \otimes I_{S_{\infty}}: \mathcal{G}_{1}^{n}\left(S_{\infty}\right) \rightarrow S_{\infty}^{n}\left(S_{\infty}\right)\right\| \\
& \geq \frac{\left\|\sum_{i, j=1}^{n} e_{i j} \otimes e_{i j}\right\|_{S_{\infty}^{n}\left(S_{\infty}^{n}\right)}}{\int_{\Omega}\left\|\left(g_{i j}(\omega)\right)\right\|_{S_{\infty}^{n}} d P(\omega)} \gtrsim n^{1 / 2} .
\end{aligned}
$$

We get the upper bound by Remark 2.3(3).

TheOREM 2.12. Let $1 / p+1 / p^{\prime}=1$.

(1) If $1 \leq p<4 / 3$, then $S_{p}$ does not have $S_{1}$-type nor $S_{s}$-cotype for $2 \leq s<p^{\prime}$.

(2) If $4 / 3 \leq p<2$, then $S_{p}$ does not have $S_{r}$-type nor $S_{s}$-cotype for

$$
\frac{2 p}{4-p}<r \leq 2 \leq s<p^{\prime} .
$$

(3) If $2<p \leq 4$, then $S_{p}$ does not have $S_{r}$-type nor $S_{s}$-cotype for

$$
p^{\prime}<r \leq 2 \leq s<\frac{2 p}{4-p} .
$$

(4) If $4<p<\infty$, then $S_{p}$ does not have $S_{r}$-type for $p^{\prime}<r \leq 2$ nor $S_{\infty}$-cotype.

(5) $S_{\infty}$ does not have $S_{1}$-type nor $S_{\infty}$-cotype.

Proof. Since the formal identity $S_{p}^{n}(E) \rightarrow S_{r}^{n}(E)$ has norm $\leq n^{1 / r-1 / p}$ for $1 \leq r<p$, we get

$$
T_{r}^{o, n}\left(S_{p}\right) \geq T_{S_{p}, n}\left(S_{p}\right) n^{1 / p-1 / r} \gtrsim n^{2 / p-1 / 2-1 / r},
$$

which means $S_{p}$ does not have $S_{r}$-type for $2 / p-1 / 2-1 / r>0 \Leftrightarrow 2 p /(4-p)$ $<r$. The other statements are obtained by duality (Proposition 2.4), Theorem 2.9, Theorem 2.8 and the fact that $R[p]$ is a closed subspace of $S_{p}$.

We close this section with the case of $C^{*}$-algebras and their duals. $S_{1}$-type and $S_{\infty}$-cotype are related to subhomogeneity of a $C^{*}$-algebra.

Theorem 2.13. Let $A$ be a $C^{*}$-algebra. Then $A$ is subhomogeneous if and only if $A$ has $S_{\infty}$-cotype if and only if $A^{*}$ has $S_{1}$-type. Moreover, if $A$ 
is not subhomogeneous, then

$$
T_{1}^{o, n}\left(A^{*}\right) \sim C_{\infty}^{o, n}(A) \sim n^{1 / 2},
$$

which is worst possible.

Proof. Suppose that $A$ is $m$-subhomogeneous for some $m \in \mathbb{N}$. Then we can assume that $A^{* *}$ is a subalgebra of $L_{\infty}\left(\mu, S_{\infty}^{m}\right)$ for some measure space $(\mathcal{M}, \mu)$. Since $L_{\infty}\left(\mu, S_{\infty}^{m}\right)$ has $S_{\infty}$-cotype and $L_{1}\left(\mu, S_{1}^{m}\right)$ has $S_{1}$-type (Remark 2.10), so do $A$ and $A^{*}$, respectively.

Now we assume that $A$ is not subhomogeneous. Then for any $\varepsilon>0$ and $n \geq 1$ there are completely positive maps $\varrho: S_{\infty}^{n} \rightarrow A$ and $\sigma: A \rightarrow S_{\infty}^{n}$ such that

$$
\left\|\sigma \varrho-I_{S_{\infty}^{n}}\right\|_{\mathrm{cb}} \leq n\left\|\sigma \varrho-I_{S_{\infty}^{n}}\right\|<\varepsilon / n
$$

by Lemma 2.7 of [25] and [7]. Then

$$
\int_{\Omega}\left\|\sum_{i, j=1}^{n} g_{i j}(\omega) \varrho e_{i j}\right\|_{A} d P(\omega) \leq\|\varrho\|_{\mathrm{cb}} \int\left\|\sum_{\Omega}^{n} g_{i j=1}(\omega) e_{i j}\right\|_{S_{\infty}^{n}} d P(\omega) \lesssim n^{1 / 2}
$$

and

$$
\begin{aligned}
\left\|\left(\varrho e_{i j}\right)\right\|_{S_{\infty}^{n}(A)} & \geq\|\sigma\|_{\mathrm{cb}}^{-1}\left\|\left(\sigma \varrho e_{i j}\right)\right\|_{S_{\infty}^{n}\left(S_{\infty}^{n}\right)}=\left\|\left(\left(\sigma \varrho-I_{S_{\infty}^{n}}\right) e_{i j}+e_{i j}\right)\right\|_{S_{\infty}^{n}\left(S_{\infty}^{n}\right)} \\
& \geq\left\|\left(e_{i j}\right)\right\|_{S_{\infty}^{n}\left(S_{\infty}^{n}\right)}-\left\|\left(\left(\sigma \varrho-I_{S_{\infty}^{n}}\right) e_{i j}\right)\right\|_{S_{\infty}^{n}\left(S_{\infty}^{n}\right)} \\
& \geq n-\left\|\sigma \varrho-I_{S_{\infty}^{n}}\right\|_{\mathrm{cb}} n \geq n-\varepsilon
\end{aligned}
$$

which implies $C_{\infty}^{o, n}(A) \sim n^{1 / 2} ; T_{1}^{o, n}\left(A^{*}\right) \sim n^{1 / 2}$ is obtained by duality.

\section{Type $(p, H)$ and cotype $(q, H)$ of operator spaces}

3.1. Definitions and basic properties. We fix a subquadratic homogeneous Hilbertian operator space $H$ from now on. Now for an operator space $E$ we define $(2, H)$-summing norm of a map $v: E \rightarrow \ell_{2}$ by

$$
\pi_{2, H}(v)=\sup \left\{\frac{\left(\sum_{k}\left\|v x_{k}\right\|^{2}\right)^{1 / 2}}{\left\|\sum_{k} x_{k} \otimes e_{k}\right\|_{E \otimes_{\min } H}}\right\} .
$$

Note that the subquadraticity of $H$ ensures that $\pi_{2, H}(\cdot)$ is actually a norm (p. 82 of [20]). Also note that all results remain true for all $H$ which are completely isomorphic to a subquadratic homogeneous Hilbertian operator space allowing suitable constants.

Definition 3.1. An operator space $E$ is said to have type $(2, H)$ if there is a constant $C>0$ such that

$$
\ell(u) \leq C \pi_{2, H}\left(u^{*}\right)
$$

for all $n \in \mathbb{N}$ and $u: \ell_{2}^{n} \rightarrow E$.

$E$ is said to have cotype $(2, H)$ if there is a constant $C^{\prime}>0$ such that

$$
\ell^{*}(v) \leq C^{\prime} \pi_{2, H}(v)
$$


for all $n \in \mathbb{N}$ and $v: E \rightarrow \ell_{2}^{n}$. We denote the infima of such $C$ and $C^{\prime}$ by $T_{2, H}(E)$ and $C_{2, H}(E)$, respectively.

We give a description of the trace dual of $\pi_{2, H}$.

Proposition 3.2. For $n \in \mathbb{N}$ and $u: \ell_{2}^{n} \rightarrow E$ we have

$$
\pi_{2, H}^{*}(u)=\inf \|A\|_{\mathrm{HS}}\|B\|_{\mathrm{cb}},
$$

where the infimum is taken over all possible factorizations $u: \ell_{2}^{n} \stackrel{A}{\rightarrow} H^{*} \stackrel{B}{\rightarrow} E$.

Proof. Let $\alpha(u)$ be the infimum on the right hand side. For any $v: E \rightarrow$ $\ell_{2}^{n}$ and factorization $u: \ell_{2}^{n} \stackrel{A}{\rightarrow} H^{*} \stackrel{B}{\rightarrow} E$ we have

$$
\begin{aligned}
|\operatorname{tr}(v u)| & \leq|\operatorname{tr}(v B A)|=|\operatorname{tr}(A v B)| \leq\|A\|_{\mathrm{HS}}\|v B\|_{\mathrm{HS}} \\
& =\|A\|_{\mathrm{HS}} \pi_{2, H}(v B) \leq\|A\|_{\mathrm{HS}}\|B\|_{\mathrm{cb}} \pi_{2, H}(v),
\end{aligned}
$$

which implies $\pi_{2, H}^{*}(u) \leq \alpha(u)$.

For the opposite inequality we will show that $\alpha^{*}(v) \geq \pi_{2, H}(v)$ for any $v: E \rightarrow \ell_{2}^{n}$. For any given $\varepsilon>0$ we choose $\left(x_{k}\right) \subseteq E$ such that

$$
\left(\sum_{k}\left\|v x_{k}\right\|^{2}\right)^{1 / 2} \geq(1-\varepsilon)\left\|\sum_{k} x_{k} \otimes e_{k}\right\|_{E \otimes_{\min } H} .
$$

Then there is $\left(y_{k}^{*}\right) \in \ell_{2}^{n}\left(\ell_{2}^{n}\right)$ with norm 1 such that

$$
\left|\left\langle\left(y_{k}^{*}\right),\left(v x_{k}\right)\right\rangle\right| \geq(1-\varepsilon)^{2}\left\|\left(v x_{k}\right)\right\|_{\ell_{2}^{n}\left(\ell_{2}^{n}\right)}=(1-\varepsilon)^{2}\left(\sum_{k}\left\|v x_{k}\right\|^{2}\right)^{1 / 2} .
$$

Consider $A: \ell_{2}^{n} \rightarrow H_{n}^{*}, z \mapsto\left(\left\langle y_{k}^{*}, z\right\rangle\right)_{k}$, and $B: H_{n}^{*} \rightarrow E, e_{k} \mapsto x_{k}$. Then

$$
\begin{aligned}
\alpha^{*}(v) & \geq \frac{|\operatorname{tr}(v B A)|}{\|A\|_{\mathrm{HS}}\|B\|_{\mathrm{cb}}}=\frac{|\operatorname{tr}(A v B)|}{\left\|\sum_{k} x_{k} \otimes e_{k}\right\|_{E \otimes_{\min } H}} \\
& =\frac{\left|\sum_{k}\left\langle A^{*} e_{k}, v B e_{k}\right\rangle\right|}{\left\|\sum_{k} x_{k} \otimes e_{k}\right\|_{E \otimes_{\min } H}}=\frac{\left|\left\langle\left(y_{k}^{*}\right),\left(v x_{k}\right)\right\rangle\right|}{\left\|\sum_{k} x_{k} \otimes e_{k}\right\|_{E \otimes_{\min } H}} \\
& \geq(1-\varepsilon)^{2} \pi_{2, H}(v) .
\end{aligned}
$$

Now we consider the $k$ th cb-approximation number of $T: E \rightarrow F$ defined by

$$
a_{k}^{o}(T):=\inf \left\{\|T-S\|_{\mathrm{cb}}: S \in C B(E, F), \operatorname{rk}(S)<k\right\} .
$$

See [15] for operator space versions of Gelfand and Kolmogorov numbers.

Proposition 3.3. For $u: H_{n}^{*} \rightarrow E$ we have

$$
\left(\sum_{k} a_{k}^{o}(u)^{2}\right)^{1 / 2} \leq \pi_{2, H}^{*}(u) .
$$


Proof. By Proposition 3.2 for any given $\varepsilon>0$ we have a factorization

$$
u: H_{n}^{*} \stackrel{A}{\rightarrow} H^{*} \stackrel{B}{\rightarrow} E
$$

with $\|A\|_{\mathrm{HS}}\|B\|_{\mathrm{cb}} \leq(1+\varepsilon) \pi_{2, H}^{*}(u)$. Thus,

$$
\begin{aligned}
\left(\sum_{k} a_{k}^{o}(u)^{2}\right)^{1 / 2} & =\left(\sum_{k} a_{k}^{o}(B A)^{2}\right)^{1 / 2} \leq\|B\|_{\mathrm{cb}}\left(\sum_{k} a_{k}^{o}(A)^{2}\right)^{1 / 2} \\
& =\|B\|_{\mathrm{cb}}\|A\|_{\mathrm{HS}} \leq(1+\varepsilon) \pi_{2, H}^{*}(u) .
\end{aligned}
$$

Recall that there is a constant $C>0$ such that

$$
\left(\sum_{k} a_{k}(u)^{q}\right)^{1 / q} \leq \pi_{q, 2}(u) \leq \frac{C q}{q-2}\left(\sum_{k} a_{k}(u)^{q}\right)^{1 / q}
$$

for any $u: \ell_{2}^{n} \rightarrow X n \in \mathbb{N}$ and $2<q \leq \infty$. This equivalence and (1.1) and (1.2) lead us to the following definition.

Definition 3.4. Let $1 \leq p<2<q \leq \infty$. An operator space $E$ is said to have type $(p, H)$ if there is a constant $C>0$ such that

$$
\left(\sum_{k} a_{k}^{o}(v)^{p^{\prime}}\right)^{1 / p^{\prime}} \leq C \cdot \ell^{*}(v)
$$

for all $n \in \mathbb{N}$ and $v: E \rightarrow H_{n}$.

$E$ has cotype $(q, H)$ if there is a constant $C^{\prime}>0$ such that

$$
\left(\sum_{k} a_{k}^{o}(u)^{q}\right)^{1 / q} \leq C^{\prime} \cdot \ell(u)
$$

for all $n \in \mathbb{N}$ and $u: H_{n}^{*} \rightarrow E$. We denote the infima of such $C$ and $C^{\prime}$ by $T_{p, H}(E)$ and $C_{q, H}(E)$, respectively.

REMARK 3.5. (1) It is clear from the definition that type $(p, H)$ and cotype $(q, H)$ imply type $p$ and cotype $q$ as Banach spaces, respectively.

(2) Let

$$
S_{r}^{o}(E, F)=\left\{u \in C B(E, F) \mid\left\|\left(a_{k}^{o}(u)\right)_{k \geq 1}\right\|_{\ell_{r}}<\infty\right\}
$$

for $1 \leq r \leq \infty$. Then by the same argument in the proof of Proposition 1 in [11] we have

$$
K\left(t, u ; S_{1}^{o}(E, F), S_{\infty}^{o}(E, F)\right) \sim K\left(t,\left(a_{k}^{o}(u)\right)_{k \geq 1} ; \ell_{1}, \ell_{\infty}\right)
$$

for $t>0$, where $K\left(t, \cdot ; E_{0}, E_{1}\right)$ is the $K$-functional with respect to a compatible pair of Banach spaces $\left(E_{0}, E_{1}\right)$. Thus

$$
\left[S_{2}^{o}(E, F), S_{\infty}^{o}(E, F)\right]_{2 / q}=S_{q}^{o}(E, F)
$$

for $2<q<\infty$. When $E$ has cotype $(2, H)$ we have

$$
\left(\sum_{k} a_{k}^{o}(u)^{2}\right)^{1 / 2} \leq C_{2, H}(E) \ell(u)
$$


for all $n \in \mathbb{N}$ and $u: H_{n}^{*} \rightarrow E$, thus cotype $(q, H)$ (resp. type $\left.(p, H)\right)$ behaves well under interpolation as in the Banach space case.

As in the $S_{p}$-type and $S_{q}$-cotype case, we have the following duality results.

Proposition 3.6. Let $E$ be an operator space and $1 \leq p \leq 2$.

(1) If $E$ has type $(p, H)$, then $E^{*}$ has cotype $\left(p^{\prime}, H\right)$ with

$$
C_{p^{\prime}, H}\left(E^{*}\right) \leq T_{p, H}(E) .
$$

(2) If $E$ has cotype $\left(p^{\prime}, H\right)$ and is $K$-convex as a Banach space, then $E^{*}$ has type $(p, H)$ with

$$
T_{p, H}\left(E^{*}\right) \leq K(E) C_{p^{\prime}, H}(E) .
$$

Proof. Note that $\ell^{*}(v) \leq \ell\left(v^{*}\right)$ and $\ell\left(v^{*}\right) \leq K(X) \ell^{*}(v)$ for any Banach space $X$ and $v: \ell_{2} \rightarrow X$.

3.2. The case of homogeneous Hilbertian operator spaces. If we consider type $(p, H)$ and cotype $(q, H)$ of homogeneous Hilbertian operator spaces, then the calculation becomes simple, so that we can completely determine type and cotype in some cases. We only consider cotype, since type can be directly obtained by duality.

Let us start with the following lemma about the approximation numbers of formal identities between homogeneous Hilbertian operator spaces. Recall that the $k$ th cb-Gelfand number of $u: E \rightarrow F$ between operator spaces is defined by

$$
c_{k}^{o}(u):=\inf \left\{\left\|\left.u\right|_{S}\right\|_{\mathrm{cb}}: S \subseteq E, \operatorname{codim} S<k\right\}
$$

for $k \in \mathbb{N}$, and clearly

$$
c_{k}^{o}(u) \leq a_{k}^{o}(u) .
$$

Lemma 3.7. Let $\mathcal{H}$ and $\mathcal{H}^{\prime}$ be homogeneous Hilbertian operator spaces. Then for the $n$-dimensional formal identity $\operatorname{id}_{n}: \mathcal{H}_{n} \rightarrow \mathcal{H}_{n}^{\prime}$ we have

$$
c_{k}^{o}\left(\operatorname{id}_{n}\right)=a_{k}^{o}\left(\operatorname{id}_{n}\right)=\left\|\operatorname{id}_{n-k+1}: \mathcal{H}_{n-k+1} \rightarrow \mathcal{H}_{n-k+1}^{\prime}\right\|_{\mathrm{cb}}
$$

for $1 \leq k \leq n$.

Proof. Fix $1 \leq k \leq n$. By (3.1) it is enough to show that

$$
c_{k}^{o}\left(\operatorname{id}_{n}\right) \geq\left\|\operatorname{id}_{n-k+1}: \mathcal{H}_{n-k+1} \rightarrow \mathcal{H}_{n-k+1}^{\prime}\right\|_{\mathrm{cb}} .
$$

Now we consider any subspace $S \subseteq \mathcal{H}_{n}$ with $m:=\operatorname{dim} S=n-\operatorname{codim} S \geq$ $n-k+1$. Then there is a partial isometry $U_{S}: \ell_{2}^{m} \rightarrow \ell_{2}^{n}$ whose image is $S$ and

$$
U_{S}^{*} U_{S}=I_{\ell_{2}^{m}} .
$$

Thus, we have

$$
\begin{aligned}
\left\|\operatorname{id}_{n-k+1}\right\|_{\mathrm{cb}} & \leq\left\|\operatorname{id}_{m}: \mathcal{H}_{m} \rightarrow \mathcal{H}_{m}^{\prime}\right\|_{\mathrm{cb}} \leq\left\|U_{S}: \mathcal{H}_{m} \rightarrow \mathcal{H}_{n}^{\prime}\right\|_{\mathrm{cb}}\left\|U_{S}^{*}: \mathcal{H}_{n}^{\prime} \rightarrow \mathcal{H}_{m}^{\prime}\right\|_{\mathrm{cb}} \\
& =\left\|\left.\operatorname{id}_{n}\right|_{S}: \mathcal{H}_{n} \rightarrow \mathcal{H}_{n}^{\prime}\right\|_{\mathrm{cb}} .
\end{aligned}
$$


Proposition 3.8. Let $\mathcal{H}$ be a homogeneous Hilbertian operator space. Then $\mathcal{H}$ has cotype $(2, H)$ if and only if the formal identity id : $H^{*} \rightarrow \mathcal{H}$ is completely bounded.

Proof. The necessity part is clear from the definition. Now we suppose that $\mathcal{H}$ has cotype $(2, H)$. Then for $n \in \mathbb{N}$ and $u: H_{n}^{*} \rightarrow \mathcal{H}_{n}$ we have

$$
\sum_{k} a_{k}^{o}(u)^{2} \leq C^{2} \ell(u)^{2}
$$

where $C=C_{2, H}(\mathcal{H})$. If we set $u=\mathrm{id}_{n}: H_{n}^{*} \rightarrow \mathcal{H}_{n}$, then by Lemma 3.7 we have

$$
C^{2} n \geq \sum_{k=1}^{n}\left\|\operatorname{id}_{k}: H_{k}^{*} \rightarrow \mathcal{H}_{k}\right\|_{\mathrm{cb}}^{2} \geq \sum_{k=[n / 2]}^{n}\left\|\operatorname{id}_{k}\right\|_{\mathrm{cb}}^{2} \geq \frac{n}{2}\left\|\operatorname{id}_{[n / 2]}\right\|_{\mathrm{cb}}^{2},
$$

which means $\left\|\operatorname{id}_{[n / 2]}\right\|_{\mathrm{cb}} \leq \sqrt{2} C$ and consequently $\|$ id $: H^{*} \rightarrow \mathcal{H} \|_{\mathrm{cb}}$ is bounded.

Now we focus on $R[p]$ and $C[p]$.

Theorem 3.9. Let $1 \leq p, q \leq \infty$. Then $R[q]$ (resp. $C[q]$ ) has cotype $(s, C[p])$ (resp. $(s, R[p]))$ if and only if $|1 / p-1 / q|+1 / s \leq 1 / 2$.

Proof. Consider $u: R[p] \rightarrow R[q]$. Let $|1 / p-1 / q|=1 / r$. Then by Lemma 5.9 of [27] we have

$$
C B(R[p], R[q]) \cong S_{r}
$$

isometrically. Since cb-approximation numbers of $u$ and $\ell(u)$ are both unitarily invariant we can assume that

$$
u=\operatorname{diag}\left(u_{1}, \ldots, u_{n}\right), \quad n \in \mathbb{N},
$$

with $\left|u_{1}\right| \geq \cdots \geq\left|u_{n}\right|$ by the usual density argument.

Now we suppose $R[q]$ has cotype $(2, C[p])$ and set $u_{1}=\cdots=u_{n}=1$. Then

$$
\ell(u)=\left(\sum_{k=1}^{n}\left|u_{k}\right|^{2}\right)^{1 / 2}=n^{1 / 2}
$$

and by Lemma 3.7,

$$
\left(\sum_{k} a_{k}^{o}(u)^{s}\right)^{1 / s}=\left(\sum_{k}(n-k+1)^{s / r}\right)^{1 / s} \sim n^{1 / r+1 / s} .
$$

Consequently,

$$
\left|\frac{1}{p}-\frac{1}{q}\right|+\frac{1}{s}=\frac{1}{r}+\frac{1}{s} \leq \frac{1}{2} .
$$

For the converse we observe the following:

$$
\left(\sum_{k} a_{k}^{o}(u)^{s}\right)^{1 / s} \leq\left[\sum_{k}\left(\sum_{i \geq k}\left|u_{i}\right|^{r}\right)^{s / r}\right]^{1 / s}=\|U\|_{\ell_{s}^{n}\left(\ell_{r}^{n}\right)},
$$


where $U=\left(u_{i j}\right)_{i, j=1}^{n}$ with $u_{i j}=u_{j}$ for $j \leq i$ and $u_{i j}=0$ elsewhere. Thus, it is enough to show that

$$
\|U\|_{\ell_{s}^{n}\left(\ell_{r}^{n}\right)} \leq\left(\sum_{k=1}^{n}\left|u_{k}\right|^{2}\right)^{1 / 2}
$$

for $1 / r+1 / s=1 / 2$. Since

$$
\ell_{s}^{n}\left(\ell_{r}^{n}\right)=\left[\ell_{4}^{n}\left(\ell_{4}^{n}\right), \ell_{2}^{n}\left(\ell_{\infty}^{n}\right)\right]_{\theta}
$$

for $r \geq s$ and $\theta=1-4 / r$, and

$$
\ell_{s}^{n}\left(\ell_{r}^{n}\right)=\left[\ell_{4}^{n}\left(\ell_{4}^{n}\right), \ell_{\infty}^{n}\left(\ell_{2}^{n}\right)\right]_{\psi}
$$

for $r<s$ and $\psi=1-4 / s$, it suffices to consider the following three extremal cases: $(r, s)=(2, \infty),(\infty, 2)$ and $(4,4)$.

When $(r, s)=(2, \infty)$ or $(\infty, 2)$ this is trivial from the definition. The case $r=s=4$ is obtained from the inequality

$$
\left(\sum_{k=1}^{n} k\left|u_{k}\right|^{4}\right)^{1 / 4} \leq\left(\sum_{k=1}^{n}\left|u_{k}\right|^{2}\right)^{1 / 2},
$$

which can be proved by induction on $n$. Indeed, when $n=1$ it is trivial. Suppose that it is true for $n$; then

$$
\sum_{k=1}^{n} k\left|u_{k}\right|^{4} \leq\left(\sum_{k=1}^{n}\left|u_{k}\right|^{2}\right)^{2},
$$

and consequently

$$
\begin{aligned}
\sum_{k=1}^{n+1} k\left|u_{k}\right|^{4} & =\sum_{k=1}^{n} k\left|u_{k}\right|^{4}+(n+1)\left|u_{k+1}\right|^{4} \leq \sum_{k, l=1}^{n}\left|u_{k}\right|^{2}\left|u_{l}\right|^{2}+(n+1)\left|u_{k+1}\right|^{4} \\
& \leq \sum_{k, l=1}^{n+1}\left|u_{k}\right|^{2}\left|u_{l}\right|^{2}=\left(\sum_{k=1}^{n+1}\left|u_{k}\right|^{2}\right)^{2}
\end{aligned}
$$

since $\left|u_{k}\right|$ 's are nonincreasing.

The proof for $C[q]$ is the same.

REMARK 3.10. Since cotype $(2, H)$ is a local property, if $F$ is $\lambda$-cbrepresentable in $E$ for some $\lambda>0$ (i.e. every finite-dimensional subspace of $F$ can be $(1+\varepsilon) \lambda$-cb-embedded in $E$ for any $\varepsilon>0)$ then cotype $(2, H)$ of $E$ can be transferred to $F$. However, sometimes cotype $(2, H)$ can be transferred to an operator space related in a weaker sense. More precisely, let us say that $F$ is $\lambda$-representable in $E$ at every matrix level if for any $m \in \mathbb{N}, \varepsilon>0$ and finite-dimensional subspace $F^{\prime}$ of $F$ there is a subspace $E^{\prime} \subseteq E$ and an isomorphism $T: F^{\prime} \rightarrow E^{\prime}$ such that

$$
\left\|I_{M_{m}} \otimes T^{-1}: M_{m}\left(E^{\prime}\right) \rightarrow M_{m}\left(F^{\prime}\right)\right\|=1
$$


and

$$
\left\|I_{M_{m}} \otimes T: M_{m}\left(F^{\prime}\right) \rightarrow M_{m}\left(E^{\prime}\right)\right\| \leq(1+\varepsilon) \lambda .
$$

Then cotype $(2, H)$ of $E$ can be transferred to $F$ if $F$ is another homogeneous Hilbertian operator space.

Indeed, by Proposition 3.8 we need to check that $\left\|\mathrm{id}_{n}: H_{n}^{*} \rightarrow F_{n}\right\|_{\mathrm{cb}}$ is uniformly bounded with respect to $n \in \mathbb{N}$. Now we fix $n \in \mathbb{N}$. Then for any $\varepsilon>0$ there is $m \in \mathbb{N}$ such that

$$
\left\|\mathrm{id}_{n}: H_{n}^{*} \rightarrow F_{n}\right\|_{\mathrm{cb}} \leq(1+\varepsilon)\left\|I_{M_{n}} \otimes \mathrm{id}_{n}: M_{m}\left(H_{n}^{*}\right) \rightarrow M_{m}\left(F_{n}\right)\right\| .
$$

Now we set $F^{\prime}=F_{2 n}$ and choose $E^{\prime} \subseteq E$ and $T$ as above. Since $E^{\prime}$ is a subspace of $E$ we have

$$
\sum_{k=1}^{2 n} a_{k}^{o}(u) \leq C_{2, H}(E) \ell(u)
$$

for any $u: H_{2 n}^{*} \rightarrow E^{\prime}$. For $u=T \circ \mathrm{id}_{2 n}$ we have

$$
\ell(u) \leq(1+\varepsilon) \lambda \ell\left(\operatorname{id}_{2 n}\right)=(1+\varepsilon) \lambda \sqrt{2 n}
$$

and

$$
\sum_{k=1}^{2 n} a_{k}^{o}(u) \geq \sum_{k=1}^{2 n} a_{k}^{M_{m}}(u) \geq \sum_{k=1}^{2 n} a_{k}^{M_{m}}\left(\operatorname{id}_{2 n}\right)
$$

where

$$
\begin{aligned}
& a_{k}^{M_{m}}\left(v: E_{1} \rightarrow E_{2}\right) \\
& :=\inf \left\{\left\|I_{M_{m}} \otimes(v-w): M_{m}\left(E_{1}\right) \rightarrow M_{m}\left(E_{2}\right)\right\|: w \in B\left(E_{1}, E_{2}\right), \operatorname{rk}(w)<k\right\} .
\end{aligned}
$$

By a similar argument to that for Lemma 3.7 we get

$$
a_{k}^{M_{m}}\left(\operatorname{id}_{2 n}\right)=\left\|I_{M_{m}} \otimes \operatorname{id}_{k}: M_{m}\left(H_{2 n}^{*}\right) \rightarrow M_{m}\left(F_{2 n}\right)\right\|
$$

and consequently

$$
\left\|I_{M_{m}} \otimes \mathrm{id}_{n}: M_{m}\left(H_{n}^{*}\right) \rightarrow M_{m}\left(F_{n}\right)\right\| \leq \sqrt{2}(1+\varepsilon) \lambda .
$$

The situation as above does happen. By [19] we know that for every infinite-dimensional operator space $E$ there is a homogeneous Hilbertian operator space contained in $E^{\mathcal{U}}$, an ultrapower of $E$. It is well known that the local structure of $E^{\mathcal{U}}$ as an operator space is not the same as $E$, unlike the Banach space case. However, by a similar argument to the Banach space case we can show that $E^{\mathcal{U}}$ is $\lambda$-representable in $E$ at every matrix level for $\lambda=1$.

3.3. The case of $L_{p}$ spaces. As in the Banach space case, type $(1, H)$ and cotype $(\infty, H)$ are trivial for certain $H$.

Proposition 3.11. Every operator space has type $(1, H)$ and cotype $(\infty, H)$ for $H=R[p], C[p]$ and $R C[p]$. 
Proof. We only prove the type case since the cotype case is obtained by duality. Note that an operator space $E$ has type $(1, H)$ if and only if there is a constant $C>0$ such that

$$
\|v\|_{\mathrm{cb}} \leq C \cdot \ell^{*}(v)
$$

for any $n \in \mathbb{N}$ and $v: E \rightarrow H_{n}$.

First, we consider the case $H=R$. Since

$$
\left\|v: E \rightarrow R_{n}\right\|_{\mathrm{cb}} \leq\left\|v: \min (E) \rightarrow R_{n}\right\|_{\mathrm{cb}}=\pi_{2}\left(v: E \rightarrow \ell_{2}^{n}\right)
$$

by (1.45) of [1] it is enough to show that

$$
\pi_{2}(v) \leq \ell^{*}(v)
$$

for any $n \in \mathbb{N}$ and $v: E \rightarrow \ell_{2}^{n}$. By trace duality this is equivalent to the following well-known result (for example (3.14) of [17]):

$$
\ell(u) \leq \pi_{2}(u)
$$

for any $n \in \mathbb{N}$ and $u: \ell_{2}^{n} \rightarrow E$.

We can prove the case $H=C$ in the same way, and by combining these two results we get the cases of $H=R \cap C$ and $R+C$. Finally, we are done by interpolation.

Using Proposition 3.8 we can determine the condition for $L_{p}(1 \leq p \leq 2)$ spaces to have cotype 2 .

TheOrem 3.12. Let $1 \leq p \leq 2$ and $\mu$ be a $\sigma$-finite measure.

(1) $S_{p}$ has cotype $(2, H)$ if and only if the formal identity

$$
\text { id : } R C[p] \rightarrow H
$$

is completely bounded.

(2) $L_{p}(\mu)$ has cotype $(2, H)$ if and only if the formal identity

$$
\text { id : } R C\left[p^{\prime}\right] \rightarrow H
$$

is completely bounded.

Proof. (1) Suppose $S_{p}$ has cotype $(2, H)$. Then since $R[p], C[p] \subseteq S_{p}$ the formal identities

$$
\text { id }: C[p] \rightarrow H \quad \text { and } \quad \text { id }: R[p] \rightarrow H
$$

are completely bounded by Proposition 3.8, so that we get the desired conclusion. For the converse direction it suffices to show that $S_{p}$ has cotype $(2, R C[p])$, which is obtained from the Banach space case and the following fact (Proposition 4.2.6 in [8]):

$$
B\left(R C\left[p^{\prime}\right], S_{p}\right)=C B\left(R C\left[p^{\prime}\right], S_{p}\right) .
$$

(2) By the usual localization argument we can assume that $L_{p}(\mu)=$ $L_{p}[0,1]$. Suppose $L_{p}[0,1]$ has cotype $(2, H)$. Note that $\operatorname{Rad}_{p} \subseteq L_{p}[0,1]$ and 
$\operatorname{Rad}_{p} \cong R C[p]$ completely isomorphically, where $\operatorname{Rad}_{p}$ is the closed linear span of the classical Rademacher system $\left\{r_{i}\right\}$ in $L_{p}[0,1]$. Thus, the formal identity id : $R C\left[p^{\prime}\right] \rightarrow H$ is completely bounded by Proposition 3.8, so that we get the desired conclusion. For the converse direction it suffices to show that $L_{p}[0,1]$ has cotype $\left(2, R C\left[p^{\prime}\right]\right)$, which is obtained from the Banach space case and the following fact obtained similarly to Proposition 4.2.6 in [8]:

$$
B\left(R C[p], L_{p}[0,1]\right)=C B\left(R C[p], L_{p}[0,1]\right) .
$$

For a certain choice of $H$ we can recover the same behavior of type and cotype as in the Banach space case.

Corollary 3.13. $S_{p}(1 \leq p \leq 2)$ has type $(p, R+C)$ and cotype $(2, R+C)$, and $S_{q}(2 \leq q<\infty)$ has type $(2, R+C)$ and cotype $(q, R+C)$.

Proof. First we consider the case $1 \leq p \leq 2$. Since $S_{2}$ has type $(2, O H)$ and the formal identity

$$
\text { id }: O H \rightarrow R+C
$$

is a complete contraction, $S_{2}$ has type $(2, R+C)$. Thus, $S_{p}$ has type $(p, R+C)$ by Proposition 3.11 and complex interpolation, and cotype $(2, R+C)$ by Theorem 3.12.

The case $2 \leq q<\infty$ is obtained by duality.

\section{Applications}

4.1. Completely $(q, 2)$-summing maps and $S_{q}$-cotype. Now we present our operator space version of "generalized little Grothendieck's theorem".

TheOREM 4.1. Let $F$ be an operator space with $S_{q}$-cotype $(2 \leq q<\infty)$. Then

$$
B(C(K), F) \subseteq \Pi_{q, 2}^{o}(C(K), F) .
$$

Proof. Let $u \in B(C(K), F)$. Since $F$ has cotype $q$, we have $u \in \Pi_{r}(E, F)$ for all $q<r<\infty$ from the Banach space result (Theorem 11.14 in [2]). Thus, by a similar calculation to the proof of Theorem 11.13 in [2],

$$
\begin{aligned}
\left\|\left(u x_{i j}\right)\right\|_{S_{q}(F)} & \leq C_{q}^{o}(F)\left[\int_{\Omega}\left\|u\left(\sum_{i, j} g_{i j}(\omega) x_{i j}\right)\right\|_{F}^{2} d P(\omega)\right]^{1 / 2} \\
& \lesssim C_{q}^{o}(F) \pi_{r}(u)\left\|\left(x_{i j}\right)\right\|_{S_{2} \otimes_{\lambda} C(K)} \\
& =C_{q}^{o}(F) \pi_{r}(u)\left\|\left(x_{i j}\right)\right\|_{S_{2} \otimes_{\min } C(K)} .
\end{aligned}
$$

REMARK 4.2. (1) $S_{q}$-cotype conditions in Theorem 4.1 are essential. Indeed, for $n \geq 1,2 \leq q<p<\infty$ and the formal identity $I_{n}: \ell_{\infty}^{n} \rightarrow R_{n}[p]$ we have $\left\|I_{n}\right\|=\sqrt{n}$. As in the proof of Theorem 2.8, we get a subspace $F\left(\cong R_{n}[p] \otimes_{h} R_{n}[q]\right)$ of $S_{q}^{n}\left(R_{n}[p]\right)$ isometric to $S_{r}^{n}$ under the mapping 
$e_{1 j} \otimes e_{1 i} \mapsto e_{i j}$, where $r=2 p q /(p q+p-q)<2$. Now we have

$$
\left\|\sum_{j=1}^{n} e_{j} \otimes e_{1 j}\right\|_{S_{2}^{n} \otimes_{\min } \ell_{\infty}^{n}}=\left\|\sum_{j=1}^{n} e_{j} \otimes e_{1 j}\right\|_{\ell_{\infty}^{n}\left(S_{2}^{n}\right)}=1
$$

and

$$
\left\|\sum_{j=1}^{n} e_{1 j} \otimes e_{1 j}\right\|_{S_{q}^{n}\left(R_{n}[p]\right)}=\left\|\sum_{j=1}^{n} e_{j j}\right\|_{S_{r}^{n}}=n^{1 / r} .
$$

Consequently,

$$
\frac{\pi_{q, 2}^{o}\left(I_{n}\right)}{\left\|u_{n}\right\|} \geq n^{1 / r-1 / 2}
$$

(2) Unlike complete $p$-summing, complete $(q, 2)$-summing $(q>2)$ does not imply complete boundedness in general. Indeed, for $q>2$ and the formal identity $I_{n}: \min \ell_{2}^{n} \rightarrow O H_{n}$ we have

$$
\pi_{q, 2}^{o}\left(I_{n}\right)=\pi_{q, 2}^{o}\left(I_{O H_{n}}\right) \leq n^{1 / 4+1 / 2 q}
$$

by Lemma 2.7 of [12] and

$$
n \lesssim\left\|\min \ell_{2}^{n} \stackrel{I_{n}}{\rightarrow} O H_{n} \stackrel{I_{n}^{*}}{\rightarrow} \max \ell_{2}^{n}\right\|_{\mathrm{cb}} \leq\left\|I_{n}\right\|_{\mathrm{cb}}^{2}
$$

by Theorem 3.8 of [18]. Thus,

$$
\frac{\left\|I_{n}\right\|_{\mathrm{cb}}}{\pi_{q, 2}^{o}\left(I_{n}\right)} \gtrsim n^{1 / 4-1 / 2 q} .
$$

(3) We cannot extend Theorem 4.1 to the case of $B(H)$ instead of $C(K)$. If we take $F=O H$ then $F$ has $S_{2}$-cotype, but it is well-known that there is a completely bounded map from $B(H)$ into $O H$ which is not completely 2 -summing ([9]).

4.2. An operator space version of Maurey's extension theorem. In this section we consider an operator space version of Maurey's extension theorem and Kwapień's theorem. We fix a perfectly Hilbertian operator space $H$.

TheOREM 4.3. Let $E$ and $F$ be operator spaces with type $(2, H)$ and cotype $\left(2, H^{*}\right)$, respectively. Then for any subspace $G \subseteq E$ and any bounded linear map $u: G \rightarrow F$ we have an extension $\widetilde{u}: E \rightarrow F$ with

$$
\gamma_{H}(\widetilde{u}) \leq T_{2, H}(E) C_{2, H^{*}}(F)\|u\| .
$$

Proof. First we observe that we can reduce our theorem to the case where $G$ and $F$ are finite-dimensional by a standard argument. We fix $u: G \rightarrow F$ and assume that for some constant $C>0$ there are extensions

$$
u_{Z}: E \stackrel{A_{Z}}{\rightarrow} H\left(I_{Z}\right) \stackrel{B_{Z}}{\rightarrow} F \quad \text { with } \quad\left\|A_{Z}\right\|_{\mathrm{cb}} \leq 1 \quad \text { and } \quad\left\|B_{Z}\right\|_{\mathrm{cb}} \leq C\|u\|
$$

for all finite-dimensional $Z \subseteq G$. Now we consider a nontrivial ultrafilter $\mathcal{U}$ of the set of all finite-dimensional subspaces of $G$ ordered by inclusion. If we 
set

$$
A: E \rightarrow \prod_{\mathcal{U}} H\left(I_{Z}\right), \quad x \mapsto\left(A_{Z} x\right), \quad B: \overline{A(G)} \rightarrow F, \quad\left(A_{Z} x\right) \mapsto u x
$$

then

$$
\|A\|_{\mathrm{cb}} \leq \lim _{\mathcal{U}}\left\|A_{Z}\right\|_{\mathrm{cb}} \leq 1 \quad \text { and } \quad\|B\|_{\mathrm{cb}} \leq \lim _{\mathcal{U}}\left\|B_{Z}\right\|_{\mathrm{cb}} \leq C\|u\|,
$$

which leads us to the desired extension $\widetilde{u}=B P A$, where $P$ is the orthogonal projection from $\prod_{\mathcal{U}} H\left(I_{Z}\right)$ onto $\overline{A(G)}$ since the class of $H(I)$ 's for some index set $I$ is closed under ultraproduct.

Now we can assume that $G$ is finite-dimensional. Then since the range of $u$ is finite-dimensional we can assume that so is $F$. Fix $u: G \rightarrow F$, and consider any $v: F \rightarrow G$. Note that the subquadratic conditions for $H$ and $H^{*}$ together with the Remark on p. 82 of [20] enable us to use Theorem 6.1 of [20] in our situation. Thus, by Theorem 6.1 of [20] there is a factorization

$$
i v: F \stackrel{A}{\rightarrow} \ell_{2}(I) \stackrel{B}{\rightarrow} E \quad \text { with } \quad \pi_{2, H^{*}}(A) \leq 1 \quad \text { and } \quad \pi_{2, H}\left(B^{*}\right) \leq \gamma_{H}^{*}(i v),
$$

where $i: G \hookrightarrow E$ is the inclusion. If we set $\widetilde{B}=B P j$, where $P$ is the orthogonal projection from $H(I)$ onto $\overline{\operatorname{ran}(A)}$ and $j: \overline{\operatorname{ran}(A)} \hookrightarrow H(I)$ is the inclusion, then we have the factorization

$$
v: F \stackrel{A}{\rightarrow} \overline{\operatorname{ran}(A)} \stackrel{\widetilde{B}}{\rightarrow} G,
$$

so that

$$
\begin{aligned}
|\operatorname{tr}(v u)| & =|\operatorname{tr}(\widetilde{B} A u)| \leq \ell^{*}(A u) \ell(\widetilde{B}) \leq\|u\| \ell^{*}(A) \ell(B) \\
& \leq\|u\| \ell^{*}(A) T_{2, H}(E) \pi_{2, H}\left(B^{*}\right) \\
& \leq T_{2, H}(E) C_{2, H^{*}}(F)\|u\| \pi_{2, H^{*}}(A) \pi_{2, H}\left(B^{*}\right) \\
& \leq T_{2, H}(E) C_{2, H^{*}}(F)\|u\| \gamma_{H}^{*}(i v) .
\end{aligned}
$$

By applying the Hahn-Banach theorem to the functional $v \mapsto \operatorname{tr}(v u)$ we can obtain the desired extension $\widetilde{u}: E \rightarrow F$ with $\gamma_{H}(\widetilde{u}) \leq T_{2, H}(E) C_{2, H^{*}}(F)\|u\|$.

COROLlary 4.4. Every operator space with type $(2, H)$ and cotype $\left(2, H^{*}\right)$ is completely isomorphic to $H(I)$ for some index set $I$.

We end this paper with an example where different choices of $H$ 's do appear.

ExAmple 4.5. Let $1<p \leq 2$. Then $S_{p}$ has cotype $(2, R C[p])$, and for any $\sigma$-finite measure $\mu, L_{p^{\prime}}(\mu)$ has type $\left(2, R C\left[p^{\prime}\right]\right)$ by Theorem 3.12 and duality. If we apply Theorem 4.3 with $G=E=L_{p^{\prime}}(\mu)$ and $F=S_{p}$ we have

$$
B\left(L_{p^{\prime}}(\mu), S_{p}\right)=\Gamma_{R C\left[p^{\prime}\right]}\left(L_{p^{\prime}}(\mu), S_{p}\right),
$$

where $\Gamma_{H}(E, F)$ denotes the set of all completely bounded maps from $E$ to $F$ which factorize completely boundedly through $H(I)$ for some index set $I$. 
Acknowledgements. The author would like to express his thanks to the anonymous referee for his/her valuable and kind comments.

\section{References}

[1] D. P. Blecher and C. Le Merdy, Operator Algebras and Their Modules-An Operator Space Approach, Oxford Univ. Press, 2004.

[2] J. Diestel, H. Jarchow and A. Tonge, Absolutely Summing Operators, Cambridge Stud. Adv. Math., 43, Cambridge Univ. Press, Cambridge, 1995.

[3] E. Dubinsky, A. Pełczyński and H. P. Rosenthal, On Banach spaces $X$ for which $\Pi_{2}\left(\mathcal{L}_{\infty}, X\right)=B\left(\mathcal{L}_{\infty}, X\right)$, Studia Math. 44 (1972), 617-648.

[4] E. G. Effros and Z. J. Ruan, Operator Spaces, London Math. Soc. Monogr. 23, Clarendon Press, Oxford Univ. Press, New York, 2000.

[5] J. García-Cuerva and J. Parcet, Vector-valued Hausdorff-Young inequality on compact groups, Proc. London Math. Soc. 88 (2004), 796-816.

[6] -, -, Quantized orthonormal systems: A non-commutative Kwapien theorem, Studia Math. 155 (2003), 273-294.

[7] T. Huruya and J. Tomiyama, Completely bounded maps of $C^{*}$-algebras, J. Operator Theory 10 (1983), 141-152.

[8] M. Junge, Factorization theory for spaces of operators, Habilitationsschrift, Kiel, 1996.

[9] — Embedding of the operator space $\mathrm{OH}$ and the logarithmic "little Grothendieck inequality", Invent. Math. 161 (2005), 225-286.

[10] S. Kwapień, Isomorphic characterizations of inner product spaces by orthogonal series with vector valued coefficients, Studia Math. 44 (1972), 583-595.

[11] H. König and L. Tzafriri, Some estimates for type and cotype constants, Math. Ann. 256 (1981), 85-94.

[12] H. H. Lee, Weak type $(2, H)$ and weak cotype $(2, H)$ of operator spaces, Houston J. Math., to appear.

[13] B. Maurey, Une nouvelle caractérisation des applications $(p, q)$-sommantes, École Polyt. Palaiseau, Sém. Maurey-Schwartz, 1973/74, Exp. XII.

[14] —, Un théorème de prolongement, C. R. Acad. Sci. Paris Sér. A 279 (1974), 329-332.

[15] T. Oikhberg, Direct sums of operator spaces, J. London Math. Soc. (2) 64 (2001), 144-160.

[16] J. Parcet, B-convex operator spaces, Proc. Edinburgh Math. Soc. (2) 46 (2003), 649-668.

[17] G. Pisier, The Volume of Convex Bodies and Banach Space Geometry, Cambridge Tracts in Math. 94, Cambridge Univ. Press, Cambridge, 1989.

[18] —, Sur les opérateurs factorisables par OH, C. R. Acad. Sci. Paris Sér. I Math. 316 (1993), 165-170.

[19] —, Dvoretzky's theorem for operator spaces, Houston J. Math. 22 (1996), 399-416.

[20] - , The operator Hilbert space OH, complex interpolation and tensor norms, Mem. Amer. Math. Soc. 122 (1996), no. 585.

[21] - Non-commutative vector valued $L_{p}$-spaces and completely $p$-summing maps, Astérisque 247 (1998).

[22] - Introduction to Operator Space Theory, London Math. Soc. Lecture Note Ser. 294, Cambridge Univ. Press, Cambridge, 2003.

[23] - Completely bounded maps into certain Hilbertian operator spaces, Int. Math. Res. Notices 2004, no. 74, 3983-4018. 
[24] G. Pisier and D. Shlyakhtenko, Grothendieck's theorem for operator spaces, Invent. Math. 150 (2002), 185-217.

[25] R. R. Smith, Completely bounded maps between $C^{*}$-algebras, J. London Math. Soc. (2) 27 (1983), 157-166.

[26] N. Tomczak-Jaegermann, Banach-Mazur Distances and Finite-Dimensional Operator Ideals, Pitman Monogr. Surveys Pure Appl. Math. 38, Longman Sci. Tech., 1989.

[27] Q. Xu, Embedding of $C_{q}$ and $R_{q}$ into noncommutative $L_{p}$-spaces, $1 \leq p<q \leq 2$, Math. Ann. 335 (2006), 109-131.

Department of Pure Mathematics

Faculty of Mathematics

University of Waterloo

200 University Avenue West

Waterloo, Ontario, Canada N2L 3G1

E-mail: lee.hunhee@gmail.com

hh5lee@math.uwaterloo.ca

Received April 12, 2006

Revised version January 8, 2008 